\title{
Hilbert, Duality, and the Geometrical Roots of Model Theory
}

\author{
Günther Eder*1 and Georg Schiemer ${ }^{\dagger 2}$ \\ ${ }^{1}$ University of Salzburg \\ ${ }^{2}$ University of Vienna
}

This article has been published (in a revised form) in [The Review of Symbolic Logic][ https://doi.org/10.1017/S1755020317000260]. This version is free to view and download for private research and study only. Not for re-distribution, re-sale or use in derivative works. @ Cambridge University Press.

\begin{abstract}
The article investigates one of the key contributions to modern structural mathematics, namely Hilbert's Foundations of Geometry (1899) and its mathematical roots in nineteenth-century projective geometry. A central innovation of Hilbert's book was to provide semantically minded independence proofs for various fragments of Euclidean geometry, thereby contributing to the development of the model-theoretic point of view in logical theory. Though it is generally acknowledged that the development of model theory is intimately bound up with innovations in 19th century geometry (in particular, the development of non-Euclidean geometries), so far, little has been said about how exactly modeltheoretic concepts grew out of methodological investigations within projective geometry. This article is supposed to fill this lacuna and investigates this geometrical prehistory of modern model theory, eventually leading up to Hilbert's Foundations.
\end{abstract}

\section{Introduction}

Nineteenth-century geometry is marked by a number of profound methodological innovations that eventually gave rise to a structural understanding of the discipline. A key example of this is the gradual development of formal axiomatics in work by e.g. Pasch and the Peanists, culminating in Hilbert's Foundations of Geometry from 1899. Hilbert's book is famous for many things. In it, Hilbert provides one

\footnotetext{
*guenther.eder@univie.ac.at

†'georg.schiemer@univie.ac.at
} 
of the first axiomatizations of Euclidean geometry that (almost) fully meets modern standards of rigor and he also presents a number of non-trivial mathematical results. But Hilbert's Festschrift is most famous not only for the mathematical content proper, but for its logico-mathematical shift in attention and its methodology: besides questions about what can be proved from the axioms of Euclidean geometry (and subsystems thereof), Hilbert for the first time in published form sets himself the task of asking questions about what cannot be logically proved from various sets of axioms. That is, he is also, and perhaps primarily, concerned with metatheoretical questions about the logical relationships between the axioms he sets up. Hilbert asks himself in a systematic way whether various systems of axioms are consistent and whether the axioms are mutually independent. So Hilbert is not just content with finding out which axioms or theorems are sufficient to prove a certain theorem, but also which axioms are necessary 1

The consistency and independence results in the Foundations are presented in a way that is with hindsight commonly described as essentially model-theoretic in character. According to this story, in order to show that a given set of axioms is consistent, Hilbert presents a model satisfying these axioms, and in order to show that an axiom or theorem is independent of a group of axioms, he presents a model where all the axioms in the group are true, but the axiom or theorem to be proved independent is false. This model-theoretic reading of Hilbert's methododology is quite common. To take a prominent example, Jaakko Hintikka, for instance, writes that 'there is no doubt that Hilbert's Foundations of Geometry was one of the main gateways of model-theoretical thinking into twentieth-century logic and philosophy' [23, p.6]. In a similar spirit, Wilfried Sieg writes that 'the (relative) consistency proofs given in Grundlagen der Geometrie are all straightforwardly semantic, using arithmetic models' [21, p. 4]. Plenty more evidence for the widespread appreciation of this view can be found in the literature. So, perhaps it is fair to say that the model-theoretic reading constitutes the received view of Hilbert's consistency and independence proofs (henceforth C \& I proofs).

The main objective of this article is to put this received view into proper perspective. In particular, we will address the following interpretive questions here: How precisely does Hilbert understand the method of modeling underling his C \& I proofs in Foundations? Are these proofs really 'straightforwardly model-theoretic' in the modern sense of the term? As we shall see, Hilbert's usage of key concepts in Foundations that are today often interpreted in a semantic way, is ambiguous. Thus, even though these concepts seem model-theoretic to a modern reader, it is not that clear whether Hilbert intended them to be understood in such a way.

In order to get a clearer picture of Hilbert's actual understanding of the method of modeling, our approach will be twofold: First, we will take a look at the geometrical prehistory of Hilbert's Foundations as well as his work leading up to it in order to see if we can find evidence for the model-theoretic reading or whether another reading is

\footnotetext{
${ }^{1}$ As Michael Hallett put it: '[...] for Hilbert, meta-mathematical investigation of a theory is as much a part of the study of a theory as is working out its consequences, or examining its foundations in the way that Frege, for instance, does.' [16, pp. 455-456]
} 
suggested. The focus here will be on the development of modern projective geometry, specifically on the debate on the proper justification of the principle of duality in projective geometry, an informal meta-theoretical principle which roughly states that the theorems in projective geometry always come in pairs. Duality is interesting in its own right, but one of the main themes of this article will be that disputes on the proper understanding and justification of the principle of duality in the 19th century reveal two basic styles of reasoning, one model-theoretic in spirit, the other proof-theoretic. 19th century mathematicians' preoccupation with duality therefore had a catalytic effect both on the development of the modern understanding of the axiomatic method and on model theory in particular. That projective geometry and the principle of duality also played a significant role for Hilbert's metatheoretical work is not only witnessed by his work leading up to the Foundations (e.g. his results on Desargues' theorem), but is evident from his famous letter to Frege:

But it is surely obvious that every theory is only a scaffolding or schema of concepts together with their necessary relations to one another, and that the basic elements can be thought of in any way one likes. If in speaking of my points I think of some system of things, e.g. the system: love, law, chimneysweep ... and then assume all my axioms as relations between these things, then my propositions, e.g. Pythagoras' theorem, are also valid for these things. In other words: any theory can always be applied to infinitely many systems of basic elements. One only needs to apply a reversible one-one transformation and lay it down that the axioms shall be correspondingly the same for the transformed things. This circumstance is in fact frequently made use of, e.g. in the principle of duality, etc., and I have made use of it in my independence proofs. (9, pp.40-41])

So a central motivation for this study of the geometrical background of the Foundations is to show that Hilbert's axiomatic approach in 1899 was not created out of the blue but rather presents an endpoint of a development towards metatheoretical reasoning in nineteenth-century geometry. Specifically, we want to show that Hilbert's method of modeling is closely connected to the different ways to justify the duality principle in projective geometry.

Second, we will take a look at how Hilbert, in his later work on the foundations of logic and mathematics in the 1920s and early 1930s, conceived of the C \& I proofs in his Foundations. Thus, the focus here will be on Hilbert's own retrospective discussions of the method of modeling at work in his early metatheoretic results. One conclusion to be drawn from this study will be that, although commentators such as Hintikka might be right in that Hilbert's proofs had 'opened the gateways to model-theoretic thinking', it is not all that clear that Hilbert himself understood his $\mathrm{C} \& \mathrm{I}$ proofs in a model-theoretic way. Furthermore, we will show that even if Hilbert's proofs are understood semantically, this does not mean that they have to be understood model-theoretically. Semantics is not necessarily model-theoretic semantics. One might well use semantic vocabulary like truth or satisfaction, without necessarily committing oneself to model-variation as a model-theoretic semantics would require. (Such an approach will be discussed in further detail in the context of Hilbert's metatheoretic results in section 4.) 
In order to substantiate these theses, we will proceed as follows: Section 2 will first investigate a particular strand in the geometrical prehistory of Hilbert's Grundlagen, namely nineteenth-century projective geometry. Following a brief discussion of the historical development of the discipline (subsection 2.1), we will focus on a key methodological debate therein, namely the debate on the proper justification of the principle of duality (subsection 2.2). Subsection 2.3 will then focus on Hilbert's own discussion of projective geometry and duality in his lecture notes from the 1890s. In section 3 we will turn to Hilbert's Foundations. Following a presentation of Hilbert's axiomatic system of Euclidean geometry (subsection 3.1), we will give a closer discussion of the actual C \& I proofs contained in the book (subsection 3.2 and discuss the main alternatives as to how these proofs can be understood (subsection 3.3). Based on this, we will then turn to Hilbert's post-Foundations work on the foundations of logic and mathematics in section 4, again searching for clues about how Hilbert intended his C \& I proofs to be understood. In the final section 5, we will try - using our findings - to substantiate the thesis already mentioned: that the classification of Hilbert as an early proponent of model-theory is not as straightforward as many scholars think it is. We will also link the emerging picture of Hilbert's C \& I proofs and its underlying account of logic and mathematics to discussions about early semantics more generally.

\section{Projective geometry and duality}

As we want to show in this paper, Hilbert's metatheoretic approach in Foundations, in particular the method of modeling underlying his independence and consistency proofs, was not developed in a vacuum, but has itself a prehistory. Various scholars in the history of mathematics have stressed the importance of the rise of non-Euclidean geometries in the work by Gauss, Bolyai, Lobachevsky, Beltrami, Klein and others in the 19th century as an important background for Hilbert's foundational work and for the development of model theory more generally: ${ }_{2}^{2}$ Less effort, however, has been spent on investigating the effects of another major branch in 19th century geometry, namely projective geometry $\left.\right|^{3}$ In order to form an accurate picture of Hilbert's Foundations and its underlying methodology, we will, therefore, first provide an overview of some of the issues that inspired projective geometry and that ultimately lead to the formation of modern projective geometry as a discipline in its own right. Although far from being comprehensive and somewhat informal in tone, this overview will nonetheless be important to get a sense of projective geometry's basic ideas and set the stage for the discussion that follows. Our main focus, then, will be on the principle of duality, how it was understood by geometers in the 19th century, and how they thought it was to be justified. As our historical discussion of the principle as well as our subsequent modern reconstructions will show, disputes over duality in the 19th century reveal two distinctive styles of reasoning, one model-theoretic

\footnotetext{
${ }^{2}$ See, e.g., 28, 36, 35, and [13.

${ }^{3}$ See 10 for a recent study of Hilbert's background in nineteenth-century geometry. For a detailed survey of the interesting pre-history of projective geometry, see [1].
} 
in spirit, the other proof-theoretic. As we will try to show in later sections, these different views on duality might provide important background for a proper understanding and evaluation of Hilbert's methodology used in the C \& I proofs of his Foundations.

\subsection{Origins of modern projective geometry}

As the term suggests, projective geometry has its roots in the study of problems concerning perspective projections in the early renaissance in the 15th century. The basic problem here is to depict an object on a sheet of paper so that the picture faithfully represents how the object is seen from a particular point of view, the projection center. Imagine, for instance, you sit in front of a canvas and you want to draw a perspectively correct picture of a cuboid which is located in front of you (see Fig. 1). The canvas determines a certain plane in three-dimensional space, the image plane, and the cuboid is located in the object space. The projection center is the (hypothetical) eye-point, from which you see the cuboid. Now, obviously, the picture of the cuboid agrees with the real cuboid in some respects, while it disagrees with it in others. For instance, vertices of the real cuboid correspond to vertices in the picture and the straight lines determined by the sides of the real cuboid correspond to straight lines in the picture. On the other hand, from the eye-point, some sides of the cuboid appear shorter than they are in reality. More generally, lengths and angles are not preserved when passing from the real cuboid to the picture (except for certain special cases). Moreover, and importantly, the images of two straight lines determined by certain parallel sides of the cuboid are no longer parallel, but intersect each other in a point $V$, the vanishing point.

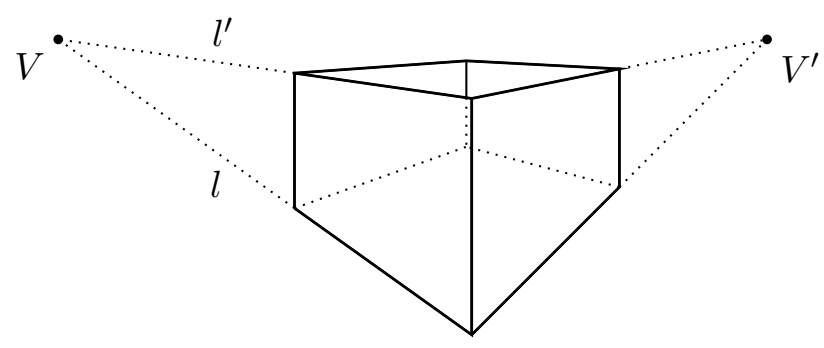

Figure 1: Cuboid in perspective with vanishing points $V$ and $V^{\prime}$.

As one might suspect from all of this, perspectively correct drawing creates all sorts of problems. Naturally, such problems attracted painters, architects, and even military engineers, and many of these people were involved in the further development of perspective drawing. Scholars back then were mostly practically oriented and mainly concerned with finding techniques that work, rather than providing rigorous proofs of why these techniques worked and how they are related to the mathematical knowledge of the time. And so, it is usually Girard Desargues (1591-1661) who is 
regarded as the father (or, perhaps, the grandfather) of projective geometry ${ }^{4}$

Like earlier scholars in the field, Desargues was an engineer and architect, and so was also to a large extent interested in practical problems relating to perspective drawing. But more than others, Desargues was also interested in the mathematical basis of the study of perspective and (at least some of the) issues that only later were clearly recognized as belonging to projective geometry. Among the most important of his works is his book Brouillon projet d'une atteinte aux evenments des recontres d'une cone avec un plan (first printed in 1639), which is generally acknowledged as one of the founding documents of projective geometry and which is mostly concerned with conic sections. Due to the simultaneous rise of Descartes' analytic geometry, Desargues' book was not widely appreciated in its day, but it does contain a number of important inventions that became important again in the 19th century. One of Desargues' foremost ideas was the introduction of so-called points at infinity. To get Desargues' idea, take for instance the straight lines $l$ and $l^{\prime}$ in Fig. 1. Suppose $l$ and $l^{\prime}$ are the images of the 'real' straight lines $g$ and $g^{\prime}$, which are determined by the two corresponding sides of the cuboid. By definition, then, the image lines $l$ and $l^{\prime}$ are images of the lines $g$ and $g^{\prime}$. But this seems to create a puzzle: Since these image lines meet in a certain point $V$ and $l$ and $l^{\prime}$ are images of $g$ and $g^{\prime}$, one would expect there to be a point 'in reality' where $g$ and $g^{\prime}$ meet, and whose image is $V$. But, of course, there is no such point. After all, $g$ and $g^{\prime}$ are parallel. Now, Desargues' central idea was to assume that, even though there might not be a real point, there is nonetheless what we would call an ideal point, where $g$ and $g^{\prime}$ meet. Parallel lines, therefore, have a certain point in common, their common point at infinity. Similarly, the different points at infinity make up a new ideal line, the line at infinity. So considerations concerning perspective drawing naturally lead to the introduction of elements at infinity 5

But there is a further reason for introducing such ideal elements, a reason that is more immediately related to pure mathematics and that made projective geometry so attractive for geometers in the 19th century. The basic idea is to use elements at infinity in order to make our mathematics 'smoother': elements at infinity allow us to state theorems in greater generality and to simplify proofs. As a case in point, let us look at an important theorem of projective geometry, first proven by (and named after) Desargues himself. In order to state the theorem, call two triangles $\triangle A B C$ and $\Delta A^{\prime} B^{\prime} C^{\prime}$ in perspective from a point $O$ if the lines determined by corresponding points of the triangles meet in a point $O$. Similarly, call two triangles in perspective from a line $o$ if corresponding sides of the triangles meet in distinct points that lie on the line $o$. What Desargues' theorem says, then, is this:

Desargues' Theorem. If two triangles $\triangle A B C$ and $\triangle A^{\prime} B^{\prime} C^{\prime}$ are in perspective from a point $O$, they are in perspective from a line o. (see Fig. 2, left)

\footnotetext{
${ }^{4}$ See 8 ] for a detailed study of Desargues' geometrical work and for further references.

${ }^{5}$ This, at least, is the standard story. For more details see e.g. [26, pp. 285 ff.]. The idea that there is a strong link between Desargues' theory of perspective and his discussion of projective methods has been opposed though by Kristi Andersen. See her [1, pp. 402-403] and [1, p. 441].
} 

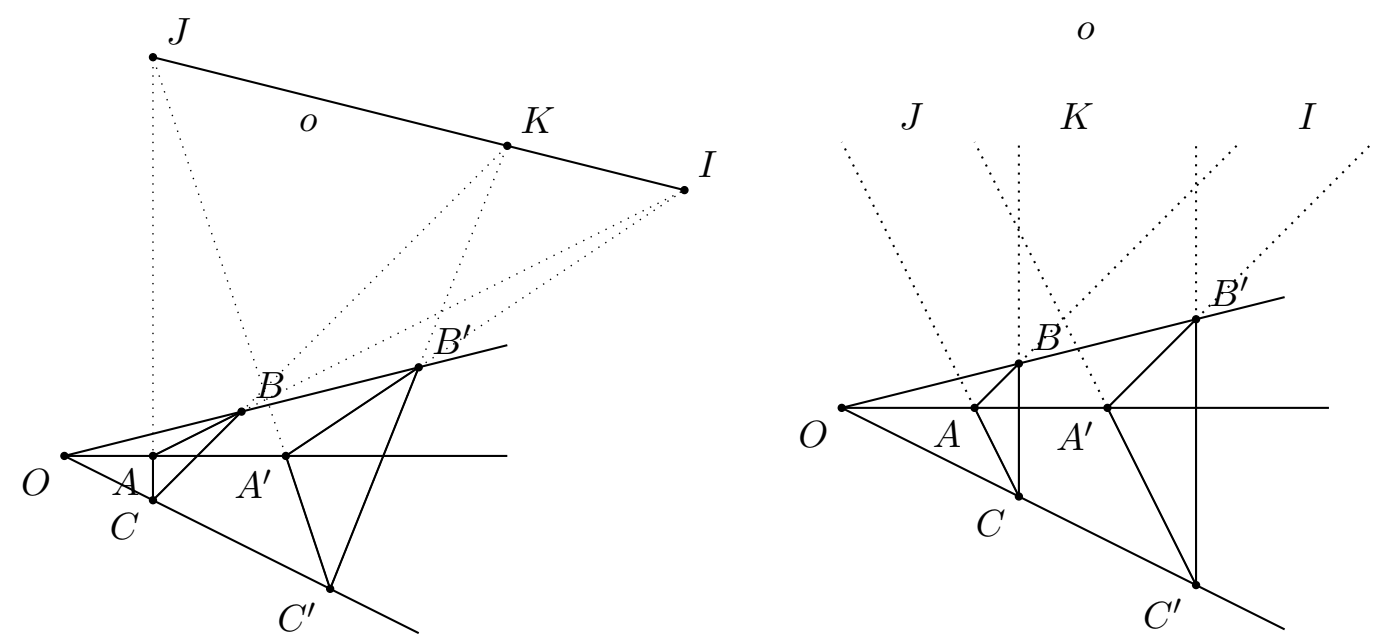

Figure 2: Two variations of the general version of Desargues' Theorem.

Now, there are all sorts of exceptions of this theorem, which are due to the fact that certain pairs of lines in the configuration are parallel. In the configuration depicted on the right-hand side of Fig. 2, for instance, the lines $A B$ and $A^{\prime} B^{\prime}$ and $A C$ and $A^{\prime} C^{\prime}$ are parallel and so the intersection points $I$ and $J$ do not exist. So if by 'points' and 'lines' we mean real points and real lines, Desargues' theorem would simply not apply, because a presupposition of the theorem is not fulfilled. However, if we allow ourselves to think of parallel lines as 'meeting at infinity' and of the points of infinity as being located on a 'line at infinity', the theorem would cover this case as well, because the theorem then simply says that if $A B$ and $A^{\prime} B^{\prime}$ and $A C$ and $A^{\prime} C^{\prime}$ are parallel, then $B C$ and $B^{\prime} C^{\prime}$ must be parallel as well. Hence, if talk about points and lines at infinity can be made sense of, we could lump together various theorems in a single, general theorem. We merely have to change our way of looking at the theorem and count ideal points and ideal lines among the things that are being talked about in the theorem. From a projective point of view, then, both configurations in Fig. 2 are in fact equivalent, and the projective version of Desargues' theorem makes a single statement about each of them indiscriminately.

The greater generality of the projective point of view also draws attention to a related advantage of projective geometry, one that was stressed by 19th century geometers. The idea is that introducing objects at infinity can often help us to prove things more smoothly or in a more 'revealing' way. Take again Desargues' theorem: In order to prove all the variations of Desargues' theorem (arising from various pairs of lines being parallel) within Euclidean geometry, we would have to prove each of them separately. Clearly, it would be more efficient if we could prove all of them in one fell swoop. And, indeed, we can, if we think of these various theorems projectively. For a typical proof of Desargues' theorem using the 'projective method', we only have to observe that if a configuration of (real or ideal) points and lines can be transformed into another configuration such that incidence relations are preserved, 
then every incidence-theorem about the first configuration is true of the second configuration and vice versa. Here, by incidence we mean the relation of a point lying on a straight line. An incidence-preserving transformation is a mapping that maps points to points and lines to lines in such a way that the image of a point $A$ lies on the image of line $l$ whenever $A$ lies on $l$, and an incidence-theorem is simply one that is formulated using only the incidence relation. Now, given this observation, there is an easy way to prove Desargues' theorem in its general form by proving a special case. Given some configuration as in Desargues' theorem, we simply 'project' the points of intersection to infinity, and thereby transform the configuration into one where the intersection points are points at infinity (like on the right-hand side of Fig. 2). What has to be proved, then, is that if two triangles are in perspective from a point $O$ and two pairs of corresponding sides of the triangles are parallel, then the third pair is parallel as well. Proving this, however, is an easy exercise in elementary Euclidean geometry ${ }^{6}$

It should be apparent already from this simple example that projective methods have the potential to greatly facilitate the systematization of geometry, and it is this feature that made projective geometry so attractive for 19th century geometers. As we mentioned earlier, Desargues' influence on his contemporaries was limited and so it was only during the late 18th, early 19th century that his ideas were rediscovered by a new generation of French geometers. This rediscovery was to a large extent effected by Gaspard Monge, but it is his pupil Jean Victor Poncelet, who is usually credited with the honorific title 'father of modern projective geometry' 7 If one had to choose the single most important book in the development of modern projective geometry, most historians of mathematics would probably cite Poncelet's Traité des propriétés projectives des figures of 1822 . Though obscure in some respects, the book contains a number of ideas that were formative for 19th century projective geometry.

Poncelet's general vision was, in the first place, evoked by a single question: Why is it, he asked himself, that analytic geometry and its algebraic methods have been that much more successful than the 'old' synthetic geometry, handed to us by the ancient Greeks? Poncelet also had an answer to this question. 'Algebra', he finds, 'employs abstract signs, it represents arbitrary magnitudes by letters which have no fixed values and which permit the magnitudes to be as undetermined as possible; consequently, algebra operates and reasons equally well on signs of non-existence as well as on signs of real quantities'. The 'non-existent' quantities that Poncelet here refers to ('creatures of the mind', [28, p. 151f]), and which stand in contrast to 'real quantities', are, of course, the negative and imaginary numbers, which had been in use for centuries and proved very useful in various areas of mathematics. So, it is the generality of algebra, its ability to cover all sorts of cases by employing 'abstract signs' that makes it superior to the old geometry. In algebra, we can allow ourselves

\footnotetext{
${ }^{6}$ Desargues himself gave two proofs of the theorem named after him, one in a purely Euclidean setting, the other using points at infinity. For more details on Desargues' theorem and its history see e.g. [26 pp. $288 \mathrm{ff}$.] and [8, pp. $161 \mathrm{ff}$.$] .$

${ }^{7}$ See, e.g., 11 .
} 
to be led by the mechanical use of signs in order to reach results. Synthetic geometry, on the other hand, though also using 'abstract signs', had always been relying on explicitly drawn diagrams ${ }^{8}$ Two paradigm cases in geometry, where, according to Poncelet, we should allow ourselves to be led by abstract considerations, are in the case of ideal elements (points and lines at infinity) and imaginary forms (more on those shortly).

We have already seen in connection with Desargues' theorem that ideal elements allow us to achieve much more generality in our formulation and proof of theorems. Poncelet, in fact, was the first to make systematic use of these ideal elements and also made a serious attempt to justify their use. ([28, p. 151]) 'It is', he claims

at bottom simply the principle of permanence or indefinite continuity of mathematical laws with respect to quantities varying insensibly, a continuity which for certain states of a given system often exists only in a purely abstract and ideal manner [...] The principle of continuity, considered simply from the point of view of geometry, consists in this, that if we suppose a given figure to change its position by having its points undergo a continuous motion without violating the conditions initially assumed to hold between them, the [...] properties which hold for the first position of the figure still hold in a generalized form for all the derived figures. (Poncelet 1822, quoted from [28, pp. 154-155])

The 'principle of continuity', as formulated here, is, to be sure, a far cry from being precise, and was attacked from various sides, including Cauchy, who called it a 'bold induction' at one point 9 But it is quite obvious how it was intended. Suppose, for instance, we start with the configuration concerning Desargues' theorem on the left-hand side of Fig. 2. By 'pulling' the vertices of the triangle in certain directions, it is easy to see that the configuration on the left-hand side can be transformed by a 'continuous motion' into the configuration on the right-hand side. Now, by the principle of continuity, then, 'the properties which hold for the first position of the figure still hold for [the derived figure]'. Because corresponding sides of both triangles of the first configuration meet in certain points that lie on a line, corresponding lines in the transformed figure must also meet in certain points that lie on a line. Of course, since the transformed lines are now parallel, these intersection points must be points at infinity and the line must be the line at infinity. So we see that the principle of continuity, in fact, implicitly postulates the existence of ideal elements, since its validity requires the existence of intersection points in the figure on the right-hand side.

As mentioned, the principle of continuity not only justifies the introduction of ideal elements, but also of 'imaginary forms', i.e. points or lines, which would be represented by complex coordinates in analytic geometry. As a paradigm case, consider two circles that meet in two points. By pulling them away from each other, we will eventually get a figure where the circles no longer intersect each other. Analytic

\footnotetext{
${ }^{8}$ As Poncelet states in the Traité: 'One always reasons upon the magnitudes themselves which are always real and existing, and one never draws conclusions which do not hold for the objects of sense, whether conceived in imagination or presented to sight.' (see [28, p. 153])

${ }^{9} \mathrm{See}$, in particular, [12, §4.2] for a more detailed discussion of Cauchy's reception of the principle.
} 
geometry's algebraic calculations, however, tell us that there are, in fact, still two points of intersection, albeit ones with complex coordinates. And we can use these imaginary points algebraically to reach results about 'real' points and lines. (For instance, as in the real case, the two imaginary points of intersection will uniquely determine a certain real line, the radical axis or power line.) So, once again, analytic geometry and its use of algebra seems to be able to account for things that synthetic geometry cannot account for. Poncelet's principle of continuity, of course, tells us another story. Since the figure we end up with is the result of a continuous movement of the points of both circles, the principle tells us that there still are two points of intersection, albeit 'invisible' ones.

\subsection{The principle of duality, informally}

A further topic that greatly exercised Poncelet and his contemporaries was the issue of duality. Duality is a striking feature of projective geometry. Roughly, it amounts to the fact that, in a projective setting, every theorem induces another theorem by simply interchanging the words 'point' and 'line' and, accordingly, the relation of a point lying on a line by the relation of a line going through a point.

In order to see duality in action, let us once again look at the projective version of Desargues' theorem. To repeat, Desargues' theorem states that if the lines that join corresponding vertices of two triangles are concurrent (meet in a point), then the intersection points of corresponding lines of the triangles are collinear (lie on a line). In short, if two triangles are in perspective from a point, then they are perspective from a line. Now, what happens when we dualize the theorem? To see this, we first dualize the vocabulary which is used to formulate the theorem:

\begin{tabular}{l|l}
\hline term & dual term \\
\hline being a point & being a line \\
lying on a line & going through a point \\
being collinear & being concurrent \\
being a triangle & being a triangle \\
\hline
\end{tabular}

Moreover, it follows that 'being in perspective from a point' is interchanged with 'being in perspective from a line' 10 So what we end up with is this:

Dual of Desargues' Theorem. If two triangles $\triangle A B C$ and $\triangle A^{\prime} B^{\prime} C^{\prime}$ are in perspective from a line $o$, they are in perspective from a point $O$.

So the dual of Desargues' theorem is simply its converse, and it is a theorem about the real projective plane just like Desargues' theorem itself. In fact, sometimes

\footnotetext{
${ }^{10}$ Note that in projective geometry the twin-concepts of a triangle (defined as a system of three non-collinear points) and of a 'three-side' (defined as a system of three non-concurrent lines), are in fact equivalent. It does not matter whether we define a triangle as a system of three non-collinear points, which determine three non-concurrent lines (the sides of the triangle) or whether we define it a as a system of three non-concurrent lines, which determine three non-collinear points (the vertices of the triangle). The notion of a triangle is, therefore, self-dual.
} 
the biconditional 'Two triangles are in perspective from a point if and only if they are in perspective from a line' is referred to as 'Desargues' theorem' in order to emphasis its self-dual character. Other well-known examples of such dual theorems are Pascal's theorem and its dual, Brianchon's theorem, or Menelaus' theorem and its dual, Ceva's theorem.

Now, one of the central insights of nineteenth-century geometers was that such cases of dual theorems were not happy coincidences, but rather consequences of a general principle, namely, the principle of duality:

Principle of duality. For any theorem of plane projective geometry we get another theorem of plane projective geometry by interchanging (1) the basic predicates 'point' and 'line', (2) the basic relations 'lies on a line' and 'goes through a point' and (3) accordingly, all notions that are defined from these basic notions ${ }^{11}$

A number of points of commentary concerning this principle are in order here: First, as was pointed out by Poncelet and others, the usefulness of duality lies in the fact that with each proof of a theorem in projective geometry, one immediately gets a proof of another statement of a symmetrical nature. So duality leads to more economy in our mathematics. Relatedly, a further significant feature of duality is that it has drawn attention to matters of logical form. It is plain that the dual of a statement has precisely the same form as the original statement. Furthermore, duality also makes clear that, in a sense, it is inessential what we take points and lines to be. By the principle of duality, points and lines are formally indistinguishable, since, in projective geometry, what can be proved about points can be proved about lines and vice versa. Finally, it is important to note here that, from a modern point of view, the principle of duality is clearly a 'metatheoretic' principle: it does not primarily deal with points, lines, and their relations to each other, but rather with the logical relationship between theorems about such objects.

As mentioned above, much work in modern projective geometry was dedicated not just to the further exploration of this principle, but also to its mathematical justification or explanation. Roughly speaking, one can identify three different approaches in the mathematical literature from the time on how duality is to be explained:

(i) a mapping- or transformation-based approach (Poncelet, Chasles)

(ii) an axiomatic or proof-theoretic approach (Gergonne, Pasch)

(iii) an analytic approach (Monge, Plücker)

For our purposes, the first two are the most important, and so we will focus on (i) and (ii) here 12

\footnotetext{
${ }^{11}$ There is a corresponding principle for solid projective geometry which states that for any theorem of solid projective geometry we get another theorem by interchanging the words 'point' and 'plane'. In what follows, we will focus on the principle of plane duality. Analogous points can be made for the solid case in the obvious way.

${ }^{12}$ See [12] and 28] for a closer discussion of the third approach. An early formulation of this classification of different explanations of the principle of duality can be found in Klein's Vorlesungen über Nicht-Euklidische Geometrie (1928). See again [28 for a detailed modern discussion and comparison of these approaches.
} 


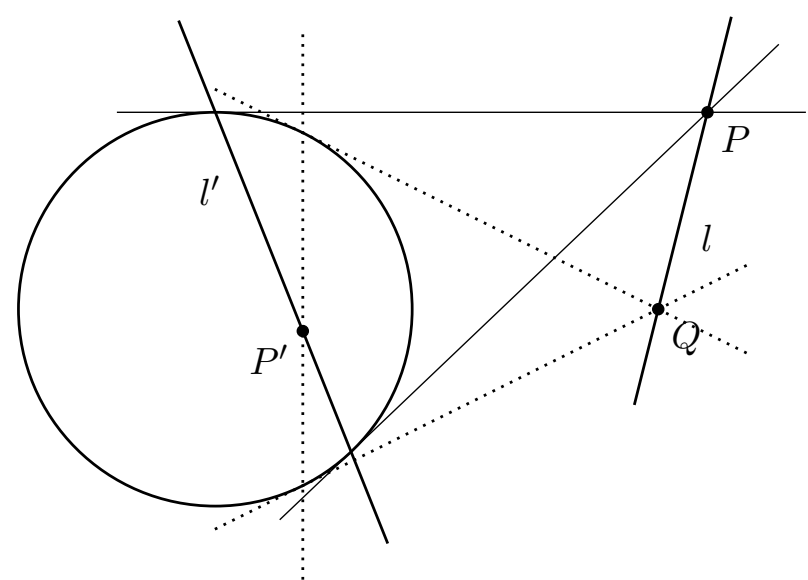

Figure 3: In the figure, the polar of $P$ is the line $l^{\prime}$, the pole of $l$ is $P^{\prime}$.

The first way to think about duality is based on transformations that preserve projective properties and can be traced back to Poncelet's Traité from 1822. More specifically, duality is discussed here in the context of the theory of poles and polars and is based on the notion of polar transformations first defined in his monograph. Roughly speaking, polars are lines that can be assigned in a systematic way to given points in the plane, relative to some conic section. Analogously, poles are points that can be assigned in a systematic way to any given line of the plane, also relative to a conic section. A polar transformation (or reciprocation) is then the systematic mapping of each point in a plane to its polar line and of each line to its corresponding pole 13

Take, for instance, the case of a circle. The polar of a given point $P$ lying outside the circle is the line $l^{\prime}$ joining the points of contact of the two tangents from $P$ to the circle. In turn, the pole of a given line $l$ outside the circle can be constructed in a similar way: given two points on $l$, say $P$ and $Q$, and tangents from $P$ and $Q$ to the circle, one can construct two lines that connect their respective points of tangency. The intersection point $P^{\prime}$ of these two lines is the pole of $l$ (see Fig. 3). The crucial property of this construction (and polar transformations more generally) is that it will guarantee the so-called reciprocity between poles and polars: if a point $P$ lies on line $l$, then the pole $P^{\prime}$ of $l$ lies on the polar line $l^{\prime}$ of $P$ (and vice versa).

Having defined these concepts in the first volume of the book, volume II of the Traité contains a section titled Théorie générale des polaires réciproques where Poncelet discusses polar reciprocal figures in the plane. In particular, Poncelet shows here that, given a figure in the plane, one can always construct a new figure (called its 'reciprocal polar') based on such a polar transformation, i.e. by a mapping of the points and lines in the figure to its polar lines and poles respectively. Such a transformation of one figure into another one has the particular property that the projective properties ('propriétés de situation') of the original figure, in particular, the incidence relations between points and lines, are preserved under this transformation. Thus, in Poncelet's own words, every projective relation between the objects of the first figure is 'immediately translated into similar relations' in the second figure [30,

\footnotetext{
${ }^{13}$ Poles and polars can be constructed geometrically and calculated analytically. See, e.g., 6] for a more textbook treatment of polar theory.
} 
p. 59].

This fact explains, according to Poncelet, why correct statements about the projective properties of a figure in the plane can be dualized in the sense stated above, viz. translated into dual statements that also turn out to be true of the newly constructed figure. The principle of duality is thus directly justified here in terms of the theory of poles and polars. It holds due to the fact that, relative to a given conic section, one can always construct a mapping between figures in the plane in which the projective properties of the figures are preserved ${ }^{14}$ Generally speaking, the informal idea underlying this mapping-based approach to duality is this: One can construct transformations between figures that leave their respective projective properties invariant. Such a structure-preserving transformation between geometrical objects can be understood to give us a translation between dual projective statements that is truth-preserving: if a statement says something correct about one figure, then its dual translation is true of the newly constructed figure. This proves the general principle of duality stated above.

The second general approach to justify the principle of duality developed in nineteenthcentury projective geometry is not semantic but rather syntactic in character. We will call it the 'axiomatic' or 'proof-theoretic' approach to duality. It is arguably rooted in work of another one of the key figures of modern projective geometry, namely Joseph Diaz Gergonne. Gergonne explicitly discusses the duality phenomenon in three articles from the 1820s published in the journal Annales de mathématiques pures et appliquées (also known as the Annales de Gergonne). The first of these articles contains the following well-known observation:

An extremely striking feature of the geometry which does not depend in any way upon metrical relations between parts of figures is that with the exception of some theorems which are themselves symmetrical [...] all the theorems are dual. That is to say, to each theorem in plane geometry there necessarily corresponds another, deduced from it by simply interchanging the two words points and lines, while in solid geometry the words points and planes must be interchanged in order to deduce the correlative from a given theorem. (Gergonne 1822, quoted from [28, p.180])

Although a systematic discussion of duality is missing in his work, there exists some textual evidence that Gergonne's justification is (proto-)axiomatic in character ${ }^{15}$ Gergonne explicitly discusses Poncelet's approach to the justification of duality in terms of polar theory at several points in his work, but eventually rejects it. The main reason for his rejection is that, unlike Poncelet, Gergonne considered duality to be a universal principle in projective geometry which is not limited to the geometry of conic sections. Polar theory cannot be used for the justification of the

\footnotetext{
${ }^{14}$ For further discussion of Poncelet's view on duality, see [28, p. 184]. Poncelet's idea of justifying duality in terms of polar transformations was taken up and significantly generalized in Michel Chasles' Aperçu historique sur l'origine et le dévéloppement des méthodes en géométrie (1837). See again [12] and 28] for detailed discussion of Chasles' work on duality.

${ }^{15}$ For a more detailed discussion of Gergonne's views on duality and its justification see [28] and [12].
} 
general duality principle since it is limited to configurations specified relative to conic sections. Gergonne's discussion of duality instead hints at an axiomatic or proof-theoretic approach: duality for him seems to be ultimately grounded in the strongly symmetrical nature of dual theorems and their proofs. More precisely, what is relevant for him is the fact that there exists a purely syntactic 'correspondence' between the primitive laws and the proofs of dual statements. In order to highlight this symmetry in logical structure, Gergonne proposes a convention that has become standard in subsequent work, namely to present dual theorems and their proofs in parallel columns.

The most explicit and extensive discussion of the axiomatic justification of duality can be found in Moritz Pasch's Vorlesungen über Neuere Geometrie (1882). As is well known, Pasch formulated the first axiomatic presentation of projective geometry in his monograph. Section 12 of the book also contains a detailed discussion of the notion of reciprocity (i.e. duality) as a property of statements about solid projective geometry. Pasch's justification of this principle given here is clearly in a similar 'proof-theoretic' spirit as the more informal remarks in Gergonne's articles. Specifically, Pasch's proof of the principle is based on two considerations: first, the fact that the axiom system presented in (1882) is strongly symmetrical in the sense that that for every axiom there exists a dual axiom of a symmetrical logical form. The second idea is what he calls the 'rigorous deductive method' at work in his treatment of axiomatic geometry. This is the assumption that all theorems of projective geometry are to be provable from the given set of axioms. Given this, duality is explained or 'verified' by him in the following way:

The law of reciprocity is first recognized to be true of the graphical sentences of $\S \S 7,8$, 9 , since the reciprocal sentence of every sentence also belongs to this group. Every other sentence to be considered here is a consequence of these sentences. In its formulation and proof only graphical concepts are used. One can restrict oneself here to the stem concepts; the other concepts are deduced from the stem concepts or can be given with the help of the relevant definitions. Every theorem is thus the result of a consideration in which only graphical stem concepts are mentioned and in which one only refers to the graphical sentences mentioned above. If one substitutes systematically the word 'point' by 'plane', 'plane' by 'point', and the used theorems by their reciprocals in this approach, then their correctness remains untouched; but as a result one finds 'point' and 'plane' interchanged, i.e. one has proved the reciprocal theorem. [29, pp. 95-96]

Note that the 'graphical sentences of $\S \S 7,8,9$ ' are basically Pasch's version of the incidence axioms for projective geometry. Pasch's argument can therefore be paraphrased as follows: The axioms of projective geometry are symmetrical in the sense that each axiom has a dual axiom. Moreover, each theorem provable from this set of axioms contains only those primitive terms specifed by the axioms or terms definable in terms of these primitive terms. The dualization of every theorem (given by the substitution of the term 'point' by 'plane', etc.) must therefore also be a theorem of the theory since it must be provable from the duals of the axioms used in the proof of the former theorem. 
Notice that this justification of duality is clearly metatheoretic in spirit, similar in this respect to the mapping-based accounts found in work by Poncelet and Chasles. However, instead of being concerned with structure-preserving transformations between geometrical systems of objects, the focus in Pasch's presentation is on the syntactic translation between geometrical statements and, most importantly, on the formal character of deductive proofs of such statements from a given set of axioms. Thus, the only thing relevant in the justification of the metatheoretic principle of duality is, in Pasch's view, the logical structure specified by the axioms and definitions of projective geometry 16

Given this background, we now want to look more closely at Hilbert's foundational work in geometry and, in particular, his views on duality.

\subsection{Hilbert on duality}

It is well known that Hilbert's foundational work in geometry, in particular his Foundations of 1899 , was strongly influenced by previous developments in axiomatic projective geometry ${ }^{17}$ Hilbert's extensive work on projective geometry is documented in detail in a number of notes for lectures held in Königsberg and Göttingen throughout the 1890s (and recently published in [20]). These lecture notes show several points of contact between his methodological approach in Foundations and the developments in projective geometry described above. In particular, they document that Hilbert's method of modeling at use in his consistency and independence proofs in [17] was first developed in the context of projective geometry, more specifically, in several proofs of the independence of Desargues' theorem from the axioms of plane projective geometry 18

A second point to be mentioned here concerns the influence of Pasch's work on Hilbert's axiomatic approach that becomes evident in these notes. There are two ideas first formulated in 29] that exercised a particularly important influence on Hilbert's work on geometry. This is, on the one hand, Pasch's deductivism, that is, the idea that geometrical proofs should no longer rely on diagrams and intuitive reasoning in general; a geometrical statement counts as a theorem only if it can be logically deduced from the axioms of the theory in question. The other influential idea is Pasch's formalism. This is, as we saw, the general idea that the intuitive interpretations we commonly attach to the basic terms of an axiomatic theory must be irrelevant in deductive proofs 19

\footnotetext{
${ }^{16}$ It should be noted here that, according to Pasch, this purely syntactic justification of the principle of duality can only be specified relative to a given axiomatization of projective geometry. In section 18 of the book, titled 'Reciprocal figures', Pasch presents a more general justification of the principle for solid projective geometry in terms of 'reciprocal or dual relations', i.e. essentially transformations closely related to the transformations in polar theory described above. See [29], p. 142].

${ }^{17}$ Compare 35] and [20] on Hilbert's different lectures on geometry in the 1890s.

${ }^{18}$ See [16] and 2] for detailed discussions of these early metatheoretical results.

${ }^{19}$ In a letter to Felix Klein dated May 23, 1893, Hilbert explicitly stresses the importance of Pasch's work for the development of modern axiomatics: '[...] I think that Pasch's ingenious book is the best way to gain insight about the controversy among geometers over the axioms. Moreover,
} 
Given Hilbert's background in projective geometry, it is interesting to see how he viewed its central metatheoretic principle, namely duality. How did he think that this principle should be justified? Somewhat surprisingly, Hilbert does not have a lot to say about the principle in his lecture notes from the 1890s. The most extensive discussion of duality can be found in the first lecture on geometry titled 'Projective geometry', dated from 1891 20 Hilbert's presentation of spatial projective geometry seems clearly 'proto-axiomatic' in character and thus comparable to his presentation of Euclidean geometry in [17]: he starts his lecture by introducing the 'basic concepts' ('Grundbegriffe') as well as eight 'fundamental laws of intuition' ('Grundgesetze der Anschauung'). These basic laws are essentially the incidence axioms of solid projective geometry. They are presented symmetrically in parallel columns in precisely the way suggested by Gergonne:

Two points $A$ and $B$ determine a line $A B$ : its connecting line.

A line $a$ and a point $B$ lying outside it determine a plane $a B$ : its connecting plane.

Three points $A, B, C$ that do not lie on a line determine a plane $A B C$ : its connecting plane.

Two lines $a$ and $b$ that have a point in common lie on a plane.
Two planes $\alpha$ and $\beta$ determine a line $\alpha \beta$ : its line of intersection.

A line $a$ and a plane $\beta$ not containing it determine a point $a \beta$ : its point of intersection.

Three planes $\alpha, \beta, \gamma$ not meeting a line determine a point: its point of intersection.

Two lines $a$ and $b$ that lie on a plane have a point $a b$ in common. [20, p. 28]

Hilbert then explicitly discusses the general principle of duality for solid projective geometry on the basis of these basic laws of intuition. He first gives the following informal description of the duality phenomenon:

The way in which I have ordered the 8 sentences in pairs makes evident the principle of duality, which is of great importance and fruitfulness given that it divides the whole subject field into 2 groups of sentences, e.g. theorem 1 etc. Points and planes correspond dually. $[\ldots]$ We will see later that, generally speaking, every sentence of projective geometry finds its complement in another sentence which results immediately from the principle of duality. [20, p. 29]

What is surprising about his subsequent discussion of duality is not the statement of the general principle, but how it is to be justified according to Hilbert. In spite of this proto-axiomatic presentation of projective geometry, the justification of the principle of duality is not given in the way suggested by Pasch some years before,

the credit of having recognized the importance of the axioms about the concept 'between' belongs to Pasch as well.' (quoted from [35, pp. 44-45], translation by the authors). See 32] and 35] for a closer discussion of Pasch's work and his influence on Hilbert.

${ }^{20}$ See [35] and [21]. 
that is, in terms of a proof-theoretic argument based on the symmetric character of the axiom system. Rather, Hilbert's proof of the general principle is given in terms of Poncelet's polar theory and based on the notion of polar transformations between reciprocal figures described above. Thus, although a proof-theoretic justification of duality, based on his proto-axiomatic presentation of projective geometry, would immediately suggest itself here, Hilbert in fact takes the 'semantic route' and justifies duality in terms of such incidence-preserving transformations. ${ }^{21}$

Why did Hilbert not adopt Pasch's metatheoretic justification of duality in this lecture? The most plausible explanation for Hilbert's transformation-based justification of duality is that, at least in 1891, Hilbert was not yet fully acquainted with Pasch's axiomatic approach and his proof-theoretic account of duality. In his presentation of projective geometry Hilbert mainly follows the first book of the third edition of Reye's Geometrie der Lage from (1886). In particular, as pointed out by Haubrich, Hilbert's own proof of duality presented in the lecture is essentially Reye's. ${ }^{22}$

Notice, in particular, that for Hilbert in 1891, the basic laws of projective geometry are not yet understood as formal axioms in the sense of Pasch's 'Stammsätze' ( [29, $\S 12]$ ), but rather as laws grounded in intuition. It follows that the symmetrical character of the axiomatization given here is not (as suggested by Pasch) the reason for the principle of duality. This symmetry of axioms rather seems to be a consequence of the general principle of duality for Hilbert. Duality, in turn, has to be justified independently of this axiomatic presentation ${ }^{23}$

While Hilbert's treatment of duality at the beginning of the 1890s is thus clearly not deductivist in Pasch's sense, it should be noted that it is nonetheless closely connected with his later work on the metatheory of axiomatic systems. In particular, the idea of dual transformations between different systems of objects present in Hilbert's proof of duality in 1891 will arguably become of central importance in his consistency and independence proofs in Foundations. That Hilbert himself was aware of this connection between the duality principle and his own subsequent metatheoretic work becomes evident in the well-known passage in the letter to Frege already cited at the beginning of section 2. Here, Hilbert seems to draw an analogy between the use of such incidence-preserving mappings in the 'semantic' justification

\footnotetext{
${ }^{21}$ The sections of the lecture notes where Hilbert sketches a proof of plane duality, namely $\S 5$ 'Poles and Polars' and $\S 7$ 'Collinearity and reciprocity', are unfortunately omitted in the published version. See the editorial introduction to the lecture notes by Haubrich for a further discussion of this point [20 pp. 16-20].

${ }^{22}$ The 'absence of the influence' of Pasch on Hilbert is discussed in Haubrich's introduction to the lecture notes in [20].

${ }^{23}$ Hilbert explicitly described projective geometry as a subtype of 'geometry of intuition' ('Geometrie der Anschauung') in the notes, that is, as a geometry whose theorems are grounded in 'basic facts of intuition' (p. 21). Toepell describes Hilbert's lecture from 1891 as explicitly non-axiomatic (in the modern or Pasch's sense). Unlike in 29, it is not held by Hilbert that the theorems of projective geometry are in fact deducible from the axioms, i.e. Hilbert's 'basic laws of intuition'. See [35]. It should be also noted here that there is a second place in Hilbert's lectures notes, namley his 1899 lecture 'Elemente der Eucklidischen Geometrie', where duality is explicitly mentioned, here in an axiomatic fashion and in context of the introduction of 'ideal elements' in geometry. See [20, pp. 357-359].
} 
of the principle of duality and his use of 'univocal and reversible one-to-one transformations' between models of an axiom system in the context of his C \& I proofs. What is the precise nature of this analogy? Moreover, can one draw a similar analogy between the genuinely non-semantic or proof-theoretic justification of duality found in Pasch's work and a proof-theoretic reading of Hilbert's metatheoretic results in his Foundations of Geometry?

In order to better see the connections between the different ways to understand duality and Hilbert's method of modeling in his Foundations, we will turn to a more detailed presentation of his main results shortly. But before this, it will be useful to give a slightly modernized presentation of the mapping-based and the proof-theoretic approach to duality first. Needless to say, we do not claim that 19th century geometers stated their results using the clean terminology of our modern reconstructions. However, as our historical discussion should have made clear, we can demarcate two strands in geometers' thinking about duality in the 19th century. The modern reconstructions that follow are intended to make even clearer why these conceptions are best understood as proto-model theoretic and proto-proof theoretic in character.

\subsection{The principle of duality, reconstructed}

Just as in ordinary Euclidean geometry, in projective geometry there are two basic kinds of objects, points and lines, and the fundamental relation among both sorts of objects is incidence, i.e. the relation of a points lying on a line ${ }^{24}$ In standard logical notation we can represent these notions by means of two one-place predicates $P, L$ and a two-place predicate $I$. We call this the language of projective geometry $\mathcal{L}_{\mathrm{PG}}{ }^{25}$ The converse of the incidence relation, the containment relation $I^{*}$, may then be defined by the stipulation that the line a contains $A$ if and only if $A$ is incident with $a$. We further say that two lines meet at a point $A$ if both lines contain $A$. We call a set of points $M$ collinear if there is a line which contains each point in $M$, and we call a set of lines $N$ concurrent if there is a point which is incident with each line in $N$.

Given this setup, we are in a position to formulate statements, and, in particular, the axioms PG of projective geometry. There are various ways to axiomatize projective geometry, but the following set of postulates will be most convenient for our purposes:

(P.1) For each pair $A, B$ of distinct points there is exactly one line which contains $A$ and $B$.

(P.2) For each pair $a, b$ of distinct lines there is exactly one point which is incident with $a$ and $b$.

(P.3) There exist at least four points $A, B, C, D$ three of which are not collinear.

\footnotetext{
${ }^{24}$ In what follows, we will focus on general plane projective geometry. A complete axiomatization of the real projective plane obviously requires additional axioms.

${ }^{25}$ By thinking of lines as sets of points, we can define incidence by the membership relation.
} 
(P.4) There exist at least four lines $a, b, c, d$ three of which are not concurrent.

Notice the distinctive axiom (P.2): it postulates the existence of points of intersection for each pair of lines. In Euclidean geometry, there are parallel lines, i.e. lines which, by definition, have no points in common. But because of (P.2), this is impossible in projective geometry. Indeed, it is (P.2) that guarentees that this axiom system is strictly symmetrical in the sense already specified by Pasch: for each axiom specifying a relation between points and lines there is another axiom that specifies the reciprocal property between them.

Projective geometry, then, is simply the set of statements in the language of projective geometry that logically follow from the postulates (P.1)-(P.4). Specifically, given some specification of a suitable background logic, one can precisely define a notion of formal derivability from these geometrical axioms. We will symbolize the fact that a sentence $\varphi \in \mathcal{L}_{\mathbf{P G}}$ is formally provable from $\mathbf{P G}$ (relative to a given logical system) by $\mathbf{P G} \vdash \varphi$.

As we saw, an important concept in projective geometry is the $d u a l \varphi^{d}$ of a statement $\varphi$. For each statement $\varphi$, formulated in $\mathcal{L}_{\mathbf{P G}}$, it is defined as the $\mathcal{L}_{\mathbf{P G}}$-statement which results from $\varphi$ by replacing each occurrence of 'point' by 'line', each occurrence of 'line' by 'point' and each occurrence of 'incidence' by 'containment' ${ }^{26}$ More formally, we can think of dualization as a translation of one formula in this language into another formula of this language, where the terms 'point' and 'line' are interchanged. Thus, for any formula $\varphi$ in the language of projective geometry, let $\varphi^{d}$ be the dual formula of $\varphi$ obtained by the translation function $[\cdot]^{d}: \mathcal{L}_{\mathbf{P G}} \rightarrow \mathcal{L}_{\mathbf{P G}}$ s.t.

(i) $[P(x)]^{d}=L(x)$

(ii) $[L(x)]^{d}=P(x)$

(iii) $[I(x, y)]^{d}=I^{*}(x, y)$

where $I^{*}(x, y)$ expresses the inverse of the incidence relation, i.e. the containment relation. Complex statements that are formed by means of logical particles are translated so that the logical structure is preserved. The translation of a negation, for instance, is simply the negation of the translation; the translation of a quantified statement is the quantified translation, etc. Given these stipulations, we can then state a proof-theoretical version of the principle of duality as follows:

Principle of duality (proof-theoretic version). For every statement $\varphi$ in the language of projective geometry: if $\boldsymbol{P} \boldsymbol{G} \vdash \varphi$, then $\boldsymbol{P} \boldsymbol{G} \vdash \varphi^{d}$.

\footnotetext{
${ }^{26}$ In order to minimize the primitive concepts and to make duality even more perspicuous, Veblen and Young, in their 1938 textbook on projective geometry, use the terminology of 'being on' for both incidence and containment. So the statements just mentioned read 'Any two distinct points of a plane are on one and only one line' and 'Any two distinct lines of a plane are on one and only one point'. [37, p. 8.]
} 
So, on the proof-theoretic version, the principle of duality simply says that the dual of every theorem is also a theorem. Read in this way, the principle of duality is to be understood as a syntactic meta-theorem, a theorem about a certain theorem-preserving translation between statements of a theory. The validity of this metatheoretic principle follows from the fact that the axiom system $\mathbf{P G}$ is strictly symmetrical together with the fact that provability is purely formal, which means that logical inference rules are insensitive to the difference between, say, $L(x)$ and $P(x)$. So, as already stressed by Pasch, when we derive a theorem from a given set of axioms, only the logical structure expressed in the axioms matters, and not some specific interpretation of its primitive terms.

Turning to the mapping-based account of duality, we have to shift our attention from the syntactic to the model-theoretic understanding of geometrical theories. So suppose $\langle\mathbf{P}, \mathbf{L}, \mathbf{I}\rangle$ is an incidence-structure, i.e. a structure of the language $\mathcal{L}_{\mathbf{P G}}$ of projective geometry. Now, call an incidence-structure $\mathfrak{P}$ that satisfies the postulates (P.1)-(P.4) a projective plane. For every projective plane $\mathfrak{P}=\langle\mathbf{P}, \mathbf{L}, \mathbf{I}\rangle$ we can then define its dual plane $\mathfrak{P}^{d}$ by the stipulation that $\mathfrak{P}^{d}=\left\langle\mathbf{L}, \mathbf{P}, \mathbf{I}^{*}\right\rangle$, where $\mathbf{I}^{*}$ is again the inverse incidence-relation ${ }^{27}$ Note that an isomorphism between an incidence structure and its dual is, therefore, simply a bijective function $\pi$ that maps points to lines and lines to points such that a point $A$ is incident with a line $a$ just in case $\pi(A)$ contains $\pi(a)$. Based on this, we can now state duality (in the spirit of Poncelet and Chasles) in terms of mappings between structures as follows:

Principle of duality (semantic version). For any projective plane $\mathfrak{P}$ : if there exists an isomorphism $\pi$ between $\mathfrak{P}$ and its dual structure $\mathfrak{P}^{d}$, then for any statement $\varphi$ in the language of projective geometry: $\mathfrak{P}=\varphi$ iff $\mathfrak{P}=\varphi^{d}$.

If presented in this form, the principle of duality clearly represents a semantic metatheorem, a statement about the behaviour of dual incidence structures. Duality is thus explained here in terms of the existence of incidence-preserving mappings (isomorphisms) between a projective plane and its dual. Notice that in this formulation of duality, the syntactic notion of formal provability is replaced by the semantic notions of satisfaction (of sentences in a structure) as well as structure-preserving mappings. More importantly, the proof of the principle of duality conceived in this way is also clearly model-theoretic in character. In fact, it is based on a fundamental model-theoretic result, known as the isomorphism lemma, which states that isomorphic structures satisfy the same sentences. Given this result, the argument for the semantic version of duality is almost trivial: Suppose $\mathfrak{P}$ is a projective plane and $\mathfrak{P}^{d}$ its dual. Now, assume that $\mathfrak{P}$ and $\mathfrak{P}^{d}$ are isomorphic. Then, by the isomorphism lemma, both structures satisfy the same sentences. That is, for all statements $\varphi$ in the language of projective geometry, we have $\mathfrak{P} \models \varphi$ iff $\mathfrak{P}^{d} \models \varphi$. Given the way in which the dual structure $\mathfrak{P}^{d}$ was specified, it follows immediately that $\mathfrak{P}^{d}=\varphi$ iff $\mathfrak{P} \models \varphi^{d}$. Combined, these two results give $\mathfrak{P}=\varphi$ iff $\mathfrak{P}=\varphi^{d}$, as required.

\footnotetext{
${ }^{27}$ As will become important later on, one can think of the dual structure $\mathfrak{P}^{d}$ as being definable in $\mathfrak{P}$ based on the translation function $[\cdot]^{d}$. Model-based interpretability in this sense is discussed in detail in section 4 .
} 
In section 3.1, we will try to use these formal explications to link our discussion of projective geometry and the principle of duality to Hilbert's foundational work in his Foundations of Geometry, to which we now turn.

\section{Independence and consistency in Hilbert's Founda- tions}

As we said in the introduction, what's new in Hilbert's treatment of Euclidean geometry in his Foundations of Geometry is that, for the first time, metamathematical investigations came to the fore in mathematics. ${ }^{28}$ Hilbert depicts the task he set himself nicely in the introduction to the Foundations:

The following investigation is a new attempt to choose for geometry a simple and complete set of independent axioms and to deduce from these the most important geometrical theorems in such a manner as to bring out as clearly as possible the significance of the different groups of axioms and the scope of the conclusions to be derived from the individual axioms. [17, p. 1]

The metamathematical character of Hilbert's investigations becomes even more clear when we look at a famous letter to Frege where Hilbert explains himself as follows:

It was of necessity that I had to set up my axiomatic system: I wanted to make it possible to understand those geometrical propositions that I regard as the most important results of geometrical inquiries: that the parallel axiom is not a consequence of the other axioms, and similarly Archimedes' axiom, etc. I wanted to answer the question whether it is possible to prove the proposition that in two identical rectangles with an identical base line the sides must also be identical, or whether as in Euclid this proposition is a new postulate. I wanted to make it possible to understand and answer such questions as why the sum of the angles in a triangle is equal to two right angles and how this fact is connected with the parallel axiom. [9, pp. 38-39]

In the quoted passage, Hilbert shows his clear focus of interest, calling the independence of the axiom of parallels and the Archimedean axiom from the remaining axioms the 'most important products of geometric investigations'. So unlike earlier mathematicians who were looking to provide clear foundations for geometry (like e.g. Moritz Pasch) from which every known geometric truth could be proved, Hilbert was not primarily interested in providing such foundations (although, of course, that was a goal as well). What he was mainly concerned with was the logical linkage between the axioms. So Hilbert was no longer exclusively concerned with questions of the form: 'What kind of truths are sufficient to prove the known theorems of geometry?', but questions of the form: 'What kind of truths are necessary to prove a certain theorem of geometry?'

\footnotetext{
${ }^{28}$ The metatheoretic character of Hilbert's approach has been discussed in detail in the literature. See e.g. [14] and 36]. See 35] and 20] for detailed surveys of the different editions and translations of Hilbert's book.
} 
Now, the problem is this: When faced with a question of the form 'Does $A$ follow from $\mathbf{S}$ ?', there are two possibilities: we either have a proof of $A$ from $\mathbf{S}$ or we don't. If we have a proof, then, of course, we immediately have an answer. But if we don't, we seem to be stuck. One possibility might be that we simply have not been smart enough to find a proof yet. But if generations of clever mathematicians fail to prove $A$ from $\mathbf{S}$, we might well be justified in suspecting that $A$ does not in fact follow from $\mathbf{S}$. The problem, then, is: How can we prove that $A$ is not a consequence of $\mathbf{S}$, but instead has to be included as an additional axiom in a complete axiomatization? Hilbert's method to prove that a certain geometrical axiom or theorem is not a consequence of a given set of axioms is usually described in the following way: There are logical terms and there are non-logical terms. The non-logical terms that occur in the geometric axioms (such as 'point', 'line', 'between', etc.) are regarded as schematic terms that are capable of being reinterpreted. In order to show that an axiom $A$ cannot be proved from a given set of axioms $\mathbf{S}$, Hilbert then reinterprets the non-logical terms in $A$ and $\mathbf{S}$ in such a way that all the axioms in $\mathbf{S}$ come out true under that reinterpretation and $A$ comes out false under that reinterpretation. For this to work, Hilbert assumes that the 'background-theory', from which the reinterpretation is constructed, is consistent. So, his C \& I proofs are relative in character. The fundamental assumption underlying this methodology is, of course, that for $A$ to be provable from $\mathbf{S}$, the particular meaning of the non-logical terms in $A$ and $\mathbf{S}$ should not matter. What matters are the logical relationships between $\mathbf{S}$ and $A$, and these relationships remain the same regardless of the meanings of the non-logical terms involved ${ }^{29}$ In order to see this method in action and to get a feel for Hilbert's interests, let us look at a couple of examples from Hilbert's Foundations.

\subsection{An axiomatization of Euclidean geometry}

Hilbert starts the Foundations by laying down his axiom system and drawing some simple consequences. His axiomatization is divided into five axiom groups: the axioms of connection, the axioms of order, the axiom of parallels (which he also calls 'Euclid's axiom'), the axioms of congruence, and the axiom of continuity (Archimedes' axiom) ${ }^{30}$ The division of the axioms into the five groups is well motivated: The axioms of connection form the 'projective basis' of his system (today these axioms are referred to as incidence axioms). That is, they state the fundamental properties about the relation of a point lying on straight line or a plane and are further divided into the plane and the spatial axioms of connection. The first axiom, for instance, is a plane axiom (I.1.) and states that two distinct points $A$ and $B$ always determine a straight line $a$. The fifth axiom, a spatial axiom, states that if two points $A, B$ of a straight line $a$ lie in a plane $\alpha$, then every point of $a$ lies in $\alpha$.

\footnotetext{
${ }^{29}$ As we saw in the previous section, this formalist account of geometrical discourse was not entirely new in 1899 but can also be found in Pasch's work on the axiomatization of projective geometry in [29]. Hilbert's approach clearly differs from Pasch's, however, in Hilbert's explicit focus on different models or interpretations of the geometrical language in question.

${ }^{30}$ In the second edition, an additional axiom of continuity is included, the axiom of completeness.
} 
The axioms of order state basic truths about the ordering of points on a straight line and 'implicitly define' the notion of a point lying between two other points. Axiom II.1., for instance, states that if $A, B, C$ are points of a straight line and $B$ lies between $A$ and $C$, then $B$ lies also between $C$ and $A$. The next group contains only one axiom, the axiom of parallels, which says that for each straight line $a$ lying in a certain plane $\alpha$ and each point $A$ of this plane which does not lie on $a$, there is exactly one straight line $b$ which is parallel to $a$, i.e., a line which has no point in common with $a 31$

The axioms of the fourth group, the axioms of congruence, determine the notion of congruence of line segments and angles and it is here that the idea of measurement first enters the stage. Using the axioms stated thus far already allows one to develop a considerable portion of elementary geometry. But in order to get a complete axiomatization of our intuitive space, Hilbert introduces two further axioms. The first one, Archimedes' axiom states that, if sufficiently often repeated on a line, every line segment $A B$ exceeds the length of any previously given line segment $C D$. The last axiom, the axiom of completeness, has a somewhat anomalous status and it was included only in the second and later editions of the Foundations. Roughly, it states that the system of points, lines and planes implicitly defined by the preceding axioms cannot be extended without violating one of the remaining axioms. Hence, unlike the other axioms, it is a 'meta-axiom' and it is for this reason that it was more controversial than the other axioms ${ }^{32}$ The importance of this axiom is, as Hilbert notes, that it guarantees that we can set up a one-to-one correspondence between the points on a line segment and the real numbers. That is, it guarantees that each line and each line segment can be conceived as an isomorophic copy of the real numbers, ordered as usual.

\subsection{Independence and consistency proofs}

Immediately after presenting the axioms, Hilbert starts discussing the consistency and mutual independence of the axioms. He starts by first considering the consistency ('compatibility') of the axioms (without the axiom of completeness) and provides the first 'model' for the axioms. He says:

\footnotetext{
${ }^{31}$ Hilbert's decision to create a separate 'axiom group' for the axiom of parallels is due to its special status and is a concession to his time. Throughout the history of mathematics, dozens of mathematicians have tried to prove or refute the axiom. But only in the 19th century, various mathematicians like Gauss, Lobatschevsky, Bolyai or Beltrami established the existence of 'NonEuclidean geometries', i.e. geometries where the axiom of parallels is false. However, lacking the precision of an exact axiomatization and a methodologically clean understanding of what is at stake when we ask ourselves about the independence of the axiom of parallels, these results were still hotly debated among philosophers. This is certainly due in part to the empirical content people associated with geometry and the fact that matters of logical consequence were mixed up with matters of empirical truth.

${ }^{32}$ Metatheoretic axioms of this kind were sometimes called extremality axioms. More specifically, the axiom of completeness is a kind of maximality axiom. See, in particular, 3], for a closer discussion of Hilbert's axiom of completeness.
} 
The axioms, which we have discussed in the previous chapter and have divided into five groups, are not contradictory to one another; that is to say, it is not possible to deduce from these axioms, by any logical process of reasoning, a proposition which is contradictory to any of them. To demonstrate this, it is sufficient to construct a geometry where all of the five groups are fulfilled. [17, p. 17]

In $\S 9$, Hilbert then presents (what many scholars today would call) an 'analytical model' for the plane axioms. In fact, he mentions several models: first, he presents a 'small', countable model where the axiom of completeness is false. After discussing the countable model in some detail, he mentions two modifications. The first modification simply accounts for the spatial axioms. The second modification assumes that we take real numbers as 'coordinates' instead of the algebraic numbers in the domain $\Omega$ he uses in the first, small model. The second modification therefore also accounts for the axiom of completeness.

Now to the details. In the model for the plane axioms, points are identified with pairs $(x, y)$ of members of a set (a "Bereich") $\Omega$ of certain algebraic numbers, straight lines as ratios $(u: v: w)$ of three such numbers (not both $u=0$ and $v=0$ ), and a point $(x, y)$ is stipulated to lie on a line $(u: v: w)$ if the equation

$$
u x+v y+w=0
$$

holds. The relation of a point lying between two points is defined as one would expect. The set $\Omega$ itself, from which the 'coordinates' of the points are drawn, is defined by stipulating that 1 is in $\Omega$ and by requiring that whenever $x, y$ are numbers in $\Omega$, then $x+y, x-y, x \times y$ and $x / y($ for $y \neq 0)$ are in $\Omega$. Additionally, it is required that for any $x$ in $\Omega, \sqrt{1+x^{2}}$ is in $\Omega$ as well.

Given these stipulations, it now has to be shown that each axiom is 'fulfilled' in this 'model'. To take a simple example, let's look at axiom I.1, which says that any two points in a plane determine a straight line (a simple case that Hilbert does not even bother to consider in detail). So, according to our translation manual, we have to show that, given two distinct points $(x, y)$ and $\left(x^{\prime}, y^{\prime}\right)$ (with $x, x^{\prime}, y, y^{\prime}$ in $\Omega$ ), we can find a unique ratio $(u: v: w)$ (with $u, v, w$ in $\Omega$, not both $u=0$ and $v=0$ ), such that the equations

$$
u x+v y+w=0
$$

and

$$
u x^{\prime}+v y^{\prime}+w=0
$$

hold simultaneously. This means that we first have to show that we can always find numbers $u, v, w$ in $\Omega$, such that both equations hold, and, second, that if $u^{\prime}, v^{\prime}, w^{\prime}$ satisfy both equations as well, then $(u: v: w)=\left(u^{\prime}: v^{\prime}: w^{\prime}\right)$.

A couple of calculations do the trick: In order to show existence, note that (1) and (2) constitute a system of linear equations in three variables. We can solve this system up to one parameter by first subtracting the second equation from the first, leading to the equation 


$$
u\left(x-x^{\prime}\right)+v\left(y-y^{\prime}\right)=0
$$

Since we assume that the points $(x, y)$ and $\left(x^{\prime}, y^{\prime}\right)$ are distinct, we have either $x \neq x^{\prime}$ or $y \neq y^{\prime}$. If $x \neq x^{\prime}$, we can choose some random number $v \neq 0$ from $\Omega$ and solve the equation (3) for $u$. Finally, we calculate $w$ from either (1) or (2). Since calculating $u$ and $w$ requires only basic arithmetical operations and $v$ is assumed to be a member of $\Omega, u$ and $w$ will be members of $\Omega$ as well. If $y \neq y^{\prime}$, we can analogously choose some number $u \neq 0$ in $\Omega$, solve (3) for $v$, and calculate $w$ from (1) or (2). Again, $v$ and $w$ will be in $\Omega$. So this proves the existence claim.

Uniqueness (up to multiples of $u, v, w$ ) goes as follows: Assume we have three numbers $u^{\prime}, v^{\prime}, w^{\prime}$ in $\Omega$ that satisfy the equations

$$
u^{\prime} x+v^{\prime} y+w^{\prime}=0
$$

and

$$
u^{\prime} x^{\prime}+v^{\prime} y^{\prime}+w^{\prime}=0
$$

as well. Subtracting (5) from (4) again, we get

$$
u^{\prime}\left(x-x^{\prime}\right)+v^{\prime}\left(y-y^{\prime}\right)=0
$$

Together with (3), this implies

$$
u: v=u^{\prime}: v^{\prime}
$$

which, together with our equations (1), (2), (4), and (5), in turn implies

$$
(u: v: w)=\left(u^{\prime}: v^{\prime}: w^{\prime}\right)
$$

which was to be proved 33

Now, this is just the simplest case, and showing that the rest of the axioms of Hilbert's system are fulfilled in this model is sometimes a bit trickier. But the example already hints at the fact that careful calculation might be needed in order to verify that a particular axiom is satisfied. In our case, for instance, showing that the axiom is satisfied in the given model required us to show that various equations are solvable by numbers in $\Omega$. In general, substantial resources from our arithmetical 'background-theory' might be needed to show that a particular axiom is satisfied in the given arithmetical model.

Sometimes, properties of that background theory of real numbers are immediately conveyed to their geometric counterparts. This can be seen most succinctly in the case of Archimedes' axiom. Given our translational scheme, the fact that a 'short' line segment can always be added to itself (finitely many times) so that the result

\footnotetext{
${ }^{33}$ As mentioned above, Hilbert's Foundations does not contain this calculation. Given his presentation of the analytic model based on $\Omega$, he merely notes that '[...] given this, as one can easily see, axioms I 1-3 and IV are satisfied.' [17, §9]
} 
exceeds any previously given 'long' line segment, is a direct consequence of the corresponding principle for the real numbers. Here, Archimedes' axiom says that we can always add a small number to itself (finitely many times) so that the resulting number exceeds any previously given large number. Indeed, it is hard to say whether it's our intuitions concerning the ordering of the real numbers (or of the numbers in $\Omega$, more precisely) that constitute our acceptance of Archimedes' axiom, or whether it's our geometrical intuitions concerning line segments.

In any case, after showing that the remaining axioms are fulfilled as well, Hilbert notes that

If, in the preceding development, we had selected the domain of all real numbers instead of the domain $\Omega$, we should have obtained likewise a geometry in which all of the axioms of groups I-V are valid. For the purposes of our demonstration, however, it was sufficient to take the domain $\Omega$, containing only an enumerable set of elements. [17, §9]

The remark is important, because it shows a particular virtue of the model Hilbert provides. The model not only shows that the axioms Hilbert provides in his original 1899 monograph are consistent, but also that the axiom he later adds to his axiomatization, the axiom of completeness, is independent of the remaining axioms.

After showing the consistency of his axiom system, Hilbert goes on to establish a number of further meta-theoretical results. More specifically, he proves that the axiom system is, in a sense, 'minimal'. That is, he shows that the axioms are mutually independent, and so none of the axioms is a consequence of the remaining axioms. The general method is as before: He shows that a certain axiom is not a consequence of the remaining axioms (or a subgroup of them) by providing a model where all axioms in the group are fulfilled except for the axiom to proved independent. The first axiom dealt with in this section is the axiom of parallels. The result is quickly reviewed without much ado. Indeed, by 1899, the independence of the axiom of parallels was firmly established. So Hilbert quickly comes to more interesting questions of independence. One of them is the question of the independence of Archimedes' axiom. The proof is interesting, since it draws our attention to a crucial methodological feature of Hilbert's independence proofs more generally. So we will look at it more closely.

Unlike in the consistency proof from earlier, Hilbert now considers all of the axioms, including the spatial ones. But similar to the consistency proof before, the basic idea is to think of points as triples $(\mathrm{x}, \mathrm{y}, \mathrm{z})$ and planes as ratios $(\mathrm{u}: \mathrm{v}: \mathrm{w}: \mathrm{r})$ of 'numbers' of a certain 'complex number system' $\Omega(t)$ (again, with the proviso that $\mathrm{u}, \mathrm{v}, \mathrm{w}$ are not all zero). The lying of a point on a plane is, similar to before, stipulated to hold if the linear equation

$$
\mathrm{ux}+\mathrm{vy}+\mathrm{wz}+\mathrm{r}=0
$$

holds and straight lines are defined as intersections of (non-parallel) planes. However, the elements of $\Omega(t)$ are no longer numbers in the usual sense. Hilbert just calls them 'numbers'. Instead, elements of $\Omega(t)$ are certain real-valued functions. Our use of non-italic letters for the variables is intended to remind the reader of 
the fact that we no longer deal with 'numbers' in the ordinary sense. More specifically, the set $\Omega(t)$ is composed of all unary functions that can be obtained from the identity function $\operatorname{id}(t)=t$ by finitely many applications of the operations addition, subtraction, multiplication, division and the operation $\sqrt{1+\mathrm{w}^{2}}$ (on functions already constructed), and which are all defined pointwise (that is, the 'sum-function' $(\mathrm{c}+\mathrm{d})(t)$ is defined by $\mathrm{c}(t)+\mathrm{d}(t)$, similarly for the other operations). Note that ' 1 ' here really means the constant function $1(t)$, having 1 as value for each real number $t$. We can also define an ordering on the functions in $\Omega(t)$ by setting $\mathrm{c}<\mathrm{d}$ if there is some real number $t_{0}$ such that $\mathrm{c}(t)<\mathrm{d}(t)$ for all numbers $t$ greater than $t_{0}$. Now, the justification for calling the functions in $\Omega(t)$ 'numbers' is that, given our stipulations concerning our five operations and ordering, it can be shown that $\Omega(t)$ has various properties that the real numbers have as well. So, for instance, addition and multiplication of functions in $\Omega(t)$ are both associative and commutative, the distributive laws hold, inequalities remain inequalities if we add a function to both sides, for each function in $\Omega(t)$ there is a greater function, etc.

The important feature of this 'complex number system' (Hilbert's term for systems of objects that satisfy some, if not all, of the properties of the real numbers) is that it does not satisfy Archimedes' axiom. In order to see this, take for instance the 'number' 1: clearly, no matter how often we add the function 1 to itself, the result will always be a constant function again. So no matter how often we add 1 to itself, it will never exceed (in the sense specified earlier), say, the identity function id. There will always be a real number $t$ greater than any constant number $n$ which means that the identity function is greater than any constant function $\mathrm{n}$. But since the ordering of this 'complex number system' is directly conveyed to the ordering of the points on a straight line, in the model Hilbert specifies, the geometrical version of Archimedes' axiom must be false as well.

\subsection{The relative character of Hilbert's proofs}

Now that we've seen how Hilbert establishes his C \& I results in his Foundations, we want to get a clearer picture of Hilbert's understanding of this method. In order to do so, let's look again at Hilbert's proof of the independence of Archimedes' axiom. What is interesting about this proof is that it draws our attention to an important feature of Hilbertian independence proofs altogether, a feature that was already present (though not that obvious) in the consistency proof of the full axiom system discussed earlier. Recall that, in order to establish the consistency of the full system, Hilbert starts with a certain 'number system' $\Omega$, from which the coordinates for the 'points' and 'straight lines' were drawn. The primitive relations between these 'points' and 'straight lines' are then determined by arithmetical equations, with the variables ranging over elements of $\Omega$. The elements of $\Omega$, from which Hilbert's 'model' was constructed, were certain real numbers, and it's the properties of this 'Pythagorean field' (which is, in fact, the smallest Pythagorean field) which account for the fact that all geometrical axioms are satisfied.

In the proof of independence of Archimedes' axiom, on the other hand, real numbers are no longer (directly) involved. Instead, the objects from which the coordinates 
for the 'points' and 'straight lines' are drawn, the elements of $\Omega(t)$, are real-valued functions which Hilbert only calls 'numbers'. Hilbert calls the objects in $\Omega(t)$ 'numbers', because various operations and relations (addition, multiplication, ordering, etc.) can be defined on $\Omega(t)$ in such a way that various (though not all) properties of the 'ordinary' real numbers are satisfied. And, again, it's the properties of this abstract 'number system' $\Omega(t)$ which guarantee that all geometric axioms except Archimedes' axiom are satisfied. So what the proof of the independence of Archimedes' axiom shows very clearly is that the 'nature' of the objects from which the 'model' is constructed is irrelevant as long as we can assume that these objects satisfy various properties. In short, it's the theory of these objects that matters. In order words, the important methodological point here is that Hilbert's C \& I proofs are essentially relative in character, relative, that is, to some background theory of objects (real numbers or real-valued functions, for instance) from which the 'models' are constructed. These objects are then shown to have various other properties that can be used to establish complex theorems that correspond to axioms (or negations of axioms) of Euclidean geometry.

Hilbert, of course, is fully well aware of this relative character of his metatheoretic proofs. Immediately after presenting his consistency proof in his Foundations, he states that ' $\mathrm{f}$ ] $\mathrm{rom}$ these considerations, it follows that every contradiction resulting from our system of axioms must also appear in the arithmetic related to the domain $\Omega .^{\prime}$ ([17, p. 21]) Thus, Hilbert is aware of the fact that we have to assume the consistency of the 'arithmetic of the domain $\Omega$ ' if his proof is to establish the consistency of his axiom system for geometry. Similarly, we have to assume that the arithmetic of the domain $\Omega(t)$ is consistent in order for Hilbert's proof to establish the independence of the geometrical version of Archimedes' axiom. The arithmetical theory that Hilbert has in mind here as a background theory in his proofs is a version of the axiom system for complete ordered fields first presented in his short paper 'Über den Zahlbegriff' from 1900 [19] ${ }^{34}$ The proofs of the independence or consistency results for (fragments of) Euclidean geometry given in Foundations are therefore always formulated against the background of this theory of real numbers in which the analytical models are constructed. Thus, as Hallett notes, what is crucial about Hilbert's approach to modeling is 'the relationship between mathematical theories' [16.

Having said this, it is not immediately clear how Hilbert made use of this relation between theories in his method of modeling. In particular, how exactly does the consistency of this axiom system for arithmetic guarantee the consistency of Euclidean geometry? Apart from casual remarks (such as the one cited earlier), Hilbert does not comment in detail on the methodology applied in his C \& I proofs until about twenty years later and he makes no attempt to justify it. One of the rare explicit descriptions of his methodology can be found in Hilbert's lecture-notes from 189899 (again, without justification), where he describes it as follows: 'In order to show

\footnotetext{
${ }^{34}$ Hilbert refers to this paper in a footnote added to the above passage. Hilbert's axiom system consists of four axiom groups, namely axioms of connection, of calculation, order and continuity (including Archimedes' axiom and an axiom of completeness.)
} 
that an axiom $\mathfrak{A}$ does not follow logically from the axioms $\mathfrak{B}, \mathfrak{C}, \mathfrak{D}, \ldots$, we present a system of things in which $\mathfrak{B}, \mathfrak{C}, \mathfrak{D}, \ldots$ hold, but $\mathfrak{A}$ does not hold.' ([20, p. 306]) A second and published place in which his method of modeling in Foundations in explicitly discussed is the following passage in Hilbert's famous 'Mathematical Problems' lecture from 1900:

In geometry one can demonstrate the consistency of the axioms by constructing a suitable domain of numbers in a way such that there correspond relations between the numbers of this domain that are analogous to those of the geometrical axioms and that henceforth any contradiction in the consequences of the geometrical axioms would also have to be recognisable in the arithmetic of that number domain [...] The axioms of arithmetic are in essence nothing other than the well-known laws of calculation with addition of the axiom of continuity. I recently collected them [in Hilbert (1900a)] ([18, pp. 264-265])

Now, despite the terminology used in these passages (and similar passages in the Foundations), which to a modern reader strongly suggest a model-theoretic reading of his proofs, it is not clear that Hilbert intended his C \& I proofs as presented in his Foundations to be so understood. The two basic interpretive options are to think of these proofs as either employing a syntactic notion of relative interpretability or a semantic notion of relative interpretability. According to the latter interpretive option, the 'standard interpretation', Hilbert constructs models (in a roughly modern sense) for geometry on the assumption that we already have a model for a certain arithmetical background theory. Thus, if correct, this interpretation would make Hilbert an early proponent of the 'model-theoretic point of view in logical theory', as scholars like Hintikka think he is ${ }^{35}$ According to the former interpretive option, on the other hand, Hilbert does not really construct models (in a roughly modern sense), but instead proves, from a certain background theory of arithmetic, the translations of geometrical theorems. Thus, on this interpretation, Hilbert would not qualify as an early model theorist (even though he might have inspired others to employ 'genuine' model theoretic methods). Notice that both interpretive options are fully compatible with what Hilbert actually does in his Foundations. Instead of thinking of the kind of consistency proof discussed earlier as making use of the modern concept of reinterpreting non-logical terms (given some model for our background theory of arithmetic), we might as well think of it as employing the idea of proving the translations of geometrical axioms from our background theory of arithmetic. Prima facie, both seem to be live options and it is the purpose of the following two sections to look for evidence that bolsters either of these interpretive options.

\section{Hilbert's metatheory revisited}

Given Hilbert's metatheoretic results in his Foundations, we want to get a better picture of his use of the 'proto-semantic' terminology and his method of modeling. As we have hinted at earlier, in Hilbert's presentation of various C \& I proofs presented in the monograph as well as in the lectures leading up to it, his use of

\footnotetext{
${ }^{35}$ See e.g. 23 and 24 .
} 
words like interpretation ('Deutung') and axioms being fulfilled ('erfüllt') or valid ('gültig') with respect to some interpretation, seem to suggest a straightforward model-theoretic reading of these proofs. Further support of such a reading has traditionally been drawn from his correspondence with Frege where he claims that 'each and every theory can always be applied to infinitely many systems of basic elements' ([9, p. 40]). And indeed, our discussion of Hilbert's work on projective geometry and the principle of duality in the early 1890s also seems to favor such an interpretation of his methodology. However, such an interpretation is not forced and, in fact, faces some serious problems. As we saw, in the case of e.g. the proof of the independence of Archimedes' axiom, we can think of his proof as being based on a translation of geometrical statements into analytic geometry and on algebraic calculations. Similarly, in the case of the axiom of parallels, we can think of his sketchy proof (which we haven't discussed here) as providing a translation of geometrical statements within Euclidean geometry and proving the translations within ordinary Euclidean geometry ${ }^{36}$ However, in and of itself, this methodology does not necessarily involve anything like 'model variation'. In what follows, we shall present further evidence that indicates that a model-theoretic reading of Hilbert's results is not as straightforward as some scholars seem to suggest. There are several passages in Hilbert's lectures and published writings from the 1920s and early 1930s where he takes a backwards look at his geometric C \& I proofs and which suggest a more nuanced view. In fact, Hilbert's retrospective remarks suggest that, even 20 or 30 years later, he is ambiguous about the method of modeling used in his Foundations. Before we look at the textual evidence, let us first be more explicit about the interpretive options outlined earlier. What possibilities for reconstructing Hilbert's method are at our disposal? In what follows we shall make more precise the two main lines of understanding Hilbert's C \& I proofs mentioned in section 2.3. The first concept is essentially proof-theoretic in character. The second is straightforwardly semantic and, perhaps, captures the semantic reading advanced by Hintikka, Demopoulos, Sieg and others most accurately. However, even on a semantic reading, it is the details that matter. We will see in due course that looking at Hilbert's proofs from a semantic point of view does not in itself entail that we have to attribute to Hilbert anything like the modern model-theoretic conception of semantics. But more on that shortly.

So, first of all, recall from the previous section that Hilbert is well aware of the relative character of his $\mathrm{C} \& \mathrm{I}$ proofs, and so any reconstruction of his methodology should therefore, in some way or another, take this feature into account. In his method of modeling, axiomatic theories are never treated in isolation but always in relation to other (axiomatic) theories. That is to say, Hilbert is aware of the fact that his metatheoretic proofs establish the independence or consistency of some set of geometrical axioms only under the assumption that a certain background theory, from which his 'models' are drawn, is consistent. (In the case of Hilbert's C

\footnotetext{
${ }^{36}$ Such a translation-based understanding of Hilbert's method is also suggested by Hallett: 'The basic technique which Hilbert adopted for this investigation [i.e. his independence proofs] is that of modeling, more strictly, of translating the theory to be investigated into another mathematical theory.' [15, p. 211, our emphasis].
} 
\& I proofs in the Foundations, this background theory is typically some theory of real numbers or real-valued functions or geometry itself.) What we are looking for, then, is a general method to show that a certain theory $\mathbf{S}$ is consistent, given the consistency of another theory $\boldsymbol{T}$ (the background theory).

There are two main options of achieving relative consistency results that are compatible with how Hilbert actually presents his C \& I proofs. Both methods are based on the notion of a translation of a language $\mathcal{L}_{\mathbf{S}}$ (in which $\mathbf{S}$ is formulated) into a language $\mathcal{L}_{\mathbf{T}}$ (in which $\mathbf{T}$ is formulated). Roughly, a translation $\tau$ is a function that maps formulas of $\mathcal{L}_{\mathbf{S}}$ to formulas of $\mathcal{L}_{\mathbf{T}}$ in such a way that their logical form is preserved. Of course, in order to make the notion of a translation technically precise, we would have to be sufficiently precise about the formal languages involved. In particular, we would have to make explicit the 'background-logic' used to formulate the theories $\mathbf{S}$ and $\mathbf{T}$. Unfortunately (though understandably, given the historic situation), in his Foundations, Hilbert is not entirely explicit in this respect. However, it is usually assumed that Hilbert's axiomatization of geometry is to be formalized within some version of higher-order logic ${ }^{37}$ For this case we can flesh the basic idea out by requiring that atomic formulas that are formed by means of the basic terms of $\mathcal{L}_{\mathbf{S}}$ are 'translated' into (simple or complex) formulas of the language $\mathcal{L}_{\mathbf{T}}$. Additional clauses will then ensure that the logical form of complex formulas is preserved. For instance, the negation of a formula is translated to the negation of the translation of the formula. The conjunction of two formulas is translated to the conjunction of the translated formulas. Similarly for the other truth-functional connectives. For first-order quantifiers, there is a clause that stipulates that a quantified formula of $\mathcal{L}_{\mathbf{S}}$ is translated to a quantified formula of $\mathcal{L}_{\mathbf{T}}$, relativized to some 'domain-formula' $\delta(x)$, so that quantifiers in the translated formula only range over the objects of the 'domain' determined by $\delta(x)$. Similarly, higher-order quantifiers are restricted to 'sets' of objects satisfying the domain-formula $\delta(x){ }^{38}$

Given the notion of a translation then, how can we show that a theory $\mathbf{S}$ is consistent relative to some consistent theory $\mathbf{T}$ ?

\subsection{Consistency and syntactic interpretability}

The first way to interpret Hilbert's method eschews semantic vocabulary altogether. The basic notion here is that of a syntactic interpretation 39

Definition. A translation $\tau$ is a syntactic interpretation of $\boldsymbol{S}$ in $\boldsymbol{T}$ if for every

\footnotetext{
${ }^{37}$ Other options include, for instance, a formalization of his axiom system using resources from set theory. One might, in addition, prefer a formalization using many-sorted logic, using different sorts of variables for points, lines and planes. In any case, the general idea of a translation as discussed below should be clear enough and may be adapted for other formalizations.

${ }^{38}$ Note that by saying that a translation $\tau$ 'preserves logical form' we merely want to highlight the fact that nothing is lost in terms of logical structure when passing from a formula to its translation. Of course, since primitive predicates are generally translated to complex formulas of the target language, the translation of a formula may have additional syntactic structure. See [38] and [27] for further details on translations.

${ }^{39}$ See again 38 , and 27. for detailed studies of the syntactic interpretability of one theory into another theory.
} 
formula $\varphi$ such that $\boldsymbol{S} \vdash \varphi$, we have $\boldsymbol{T} \vdash \varphi^{\tau}$. A theory $\boldsymbol{S}$ is syntactically interpretable in $\boldsymbol{T}$ if there is a syntactic interpretation of $\boldsymbol{S}$ in $\boldsymbol{T}$.

So what is required for a theory to be interpretable in another is the existence of a certain translation $\tau$. The notions of a model, truth in a model (or satisfaction), and all the rest of the usual semantic vocabulary is irrelevant for this concept; all that matters is that the given translation preserves theoremhood. So the translation of every theorem of $\mathbf{S}$ should be provable from the axioms of the 'interpreting' theory $\mathbf{T}$. (Equivalently, one could require that the translation of every axiom of $\mathbf{S}$ has to be provable from $\mathbf{T}$ ). So how can we prove the consistency of $\mathbf{S}$ relative to the consistency of $\mathbf{T}$ ? The idea is straightforward: since $\mathbf{T}$ proves the translation of every $\mathbf{S}$-theorem, the translation of any inconsistency in $\mathbf{S}$ would be provable in $\mathbf{T}$. That is, if $\varphi \wedge \neg \varphi$ were provable from $\mathbf{S}$, then $(\varphi \wedge \neg \varphi)^{\tau}$ would be provable from $\mathbf{T}$. But since translations preserve logical form, the translation of this statement is also a contradiction, and so $\mathbf{T}$ would be inconsistent as well. Therefore, by contraposition, if $\mathbf{T}$ is consistent, then so is $\mathbf{S}$.

Now, if we interpret Hilbert as implicitly employing the notion of syntactic relative interpretability in his early metatheoretical results, Hilbert shows the relative consistency of various fragments of Euclidean geometry without using 'models' (in the modern sense of the term) or the like. For instance, Hilbert's consistency proof for Euclidean geometry establishes that, given a certain translation of the geometrical vocabulary in the language of the theory of real numbers, the translation of every axiom of Euclidean geometry can be proved from the axioms for the real numbers. Similarly, his proof that Archimedes' axiom $A$ is independent of the rest of the axioms $\mathbf{G}$, establishes that $\mathbf{G}+\neg A$ is consistent by showing that there is a translation $\tau$, such that, for every statement $\varphi$ in $\mathbf{G}+\neg A, \varphi^{\tau}$ is a theorem of the (higherorder) theory of real numbers. Thus, in this interpretation of Hilbert's method of modeling employed in Foundations, all the seemingly semantic vocabulary is systematically explained away. In particular, the notions of a semantic interpretation of a (geometrical) language and that of a sentence being true in an interpretation are replaced here by the syntactic notion of a theorem-preserving translation between two different languages 40

And indeed, Hilbert, with hindsight, does at various passages in later writings describe his method of modeling used in the Foundations in precisely this way. For instance, in their monograph Foundations of Mathematics of 1934 (which is largely based on lectures held by Hilbert in the 1920s), Hilbert and Bernays comment on the question of relative consistency proofs in geometry and physics as follows:

Now, one usually treats this problem - both in geometry and the disciplines of physicswith the method of arithmetization: The objects of a theory are represented by numbers or systems of numbers and the basic relations by equations or inequations, such that, on the basis of this translation, the axioms of the theory turn out either as arithmetical identities or provable sentences (as in geometry), or as a system of conditions whose joint

\footnotetext{
${ }^{40}$ Hallett, in several articles, interprets Hilbert's proof method underlying his C \& I results in terms of such a notion of syntactic interpretability. See [15, p. 218] and [16, pp.460-461].
} 
satisfiability can be demonstrated via arithmetical existence sentences (as in physics). This approach presupposes the validity of arithmetic, i.e. the theory of real numbers (analysis). [22, p. 3]

It is interesting to see Hilbert and Bernays struggling here. On the one hand, they are talking about objects ('Gegenstände'), representing objects of the theory by numbers and systems of numbers and joint satisfiability of systems of conditions. On the other hand, they talk about axioms being translated and translations of axioms being provable in the theory of real numbers. Such a proof-theoretic understanding of the 'method of arithmetization' is also stated more clearly in the following passage:

This then proves that the axiom system $\mathfrak{B}$ under consideration is in fact consistent: Any contradiction that arose as a conclusion from the axiom system $\mathfrak{A}$ would, of course, represent a contradiction derivable from the axiom system $\mathfrak{A}$ even though the axiom system $\mathfrak{A}$ is known to be consistent. Arithmetic (in axiomatic formulation) presents itself as such an axiom system $\mathfrak{A}$. [22, p. 19]

Given Hilbert's and Bernays' general discussion of relative consistency proofs, two points of commentary should be made here. First, it is beyond doubt that in 1899 (and unlike 1934), Hilbert did not yet have the modern proof-theoretic methods at hand to actually give a purely syntactic account of his C \& I results. In particular, Hilbert's notion of deductive proof from axioms was (similar to that of Pasch) still an informal one at the turn of the previous century ${ }^{41}$ Nevertheless, Hilbert's own ex-post discussion of the non-provability results in Foundations suggests that one way in which Hilbert conceived of his method of modeling was along proof-theoretic lines. Although the above passages in [22] are not entirely conclusive, they do suggest that Hilbert, at least with hindsight, thought of the relative consistency proofs in his Foundations as implicitly employing the notion of a syntactic interpretation. This interpretation also suggests that Hilbert's conception of the proto-semantic notions of 'satisfaction' and 'validity' used in Foundations is rather syntactic in character if viewed from a modern vantage point: for instance, when Hilbert states that a geometrical axiom is satisfied in a particular analytic model, what he really has in mind is that the arithmetical translation of that statement is provable from the axioms of analysis $\mathbf{A}$ (stated in [19]).

Second, notice that this syntactic reconstruction also connects Hilbert's metatheoretic approach in Foundations with the preceding axiomatic tradition in projective geometry, in particular with Pasch's justification of the principle of duality. Recall from section 2.2 that the general reasoning underlying Pasch's proof of duality was also genuinely proof-theoretic in character: the dual translation of any theorem in projective geometry $\mathbf{P G}$ is also a theorem due to the symmetrical character of this axiom system as well as the formal nature of deductive proofs. Indeed, we can think

\footnotetext{
${ }^{41}$ Sieg has recently suggested to distinguish between two different conceptions of axiomatics in Hilbert's work, namely structural axiomatics and formal axiomatics (cf. 33]). According to Sieg, in his Foundations, Hilbert was a clear proponent of structural axiomatics and only later developed the idea of formal axiomatics within his proof theoretic work. As we try to establish in this paper, informal proof-theoretic methods might have nonetheless informed even his early work on geometry in Foundations.
} 
of duality itself as a syntactic interpretability result: dualization is a specific translation between statements of the language of projective geometry into the language of projective geometry that preserves theoremhood. As we saw, a very similar line of metatheoretic reasoning can be ascribed to Hilbert's C \& I proofs in Foundations. If $\mathbf{E G}$ is to stand for Euclidean geometry, we can thus draw the following structural analogy between the two proof strategies:

\begin{tabular}{ll}
$\begin{array}{l}\text { Proof-theoretic conception of du- } \\
\text { ality: }\end{array}$ & $\begin{array}{l}\text { Proof-theoretic conception of C \& } \\
\text { I Proofs: }\end{array}$ \\
\hline
\end{tabular}

\begin{tabular}{|c|c|}
\hline 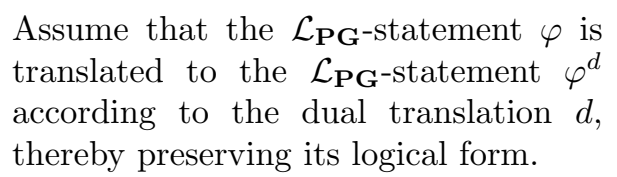 & $\begin{array}{l}\text { Assume that the } \mathcal{L}_{\mathbf{E G}} \text {-statement } \varphi \text { is } \\
\text { translated to the } \mathcal{L}_{\mathbf{A}} \text {-statement } \varphi^{\tau} \text { ac- } \\
\text { cording so a suitable translation } \tau \text {, } \\
\text { thereby preserving its logical form. }\end{array}$ \\
\hline $\begin{array}{l}\text { Then, since axioms of } \mathbf{P G} \text { are trans- } \\
\text { lated to axioms of } \mathbf{P G} \text {, if } \varphi \text { is logically } \\
\text { provable from the axioms of } \mathbf{P G}, \varphi^{d}\end{array}$ & $\begin{array}{l}\text { Then, since axioms of } \mathbf{E G} \text { are trans- } \\
\text { lated to theorems of } \mathbf{A} \text {, if } \varphi \text { is logically } \\
\text { provable from the axioms of } \mathbf{E G}, \varphi^{\tau} \\
\text { is logically provable from the axioms of } \\
\text { A. }\end{array}$ \\
\hline
\end{tabular}

According to our first reconstruction, then, Hilbert only relies on informal syntactic or proof-theoretic concepts in his C \& I proofs. The central notions are that of a translation and of provability and no use is made of genuinely semantic concepts like truth or truth in a structure, just as in the proof-theoretic justification of the (proof-theoretic version of) the principle of duality.

\subsection{Consistency and semantic interpretability}

A second possible approach to interpret Hilbert's metatheoretic proofs in Foundations is model-theoretic and is based on the notion of semantic interpretability of one theory in another theory. Before we can give a definition of this notion, notice that, given a translation $\tau$ of $\mathcal{L}_{\mathbf{S}}$ in $\mathcal{L}_{\mathbf{T}}$, we can associate with each $\mathcal{L}_{\mathbf{T}^{-}}$-structure $\mathfrak{M}$ a certain $\mathcal{L}_{\mathrm{S}^{-}}$-structure $\mathfrak{M}^{\tau}$ by letting the domain of $\mathfrak{M}^{\tau}$ be the set of objects in the domain of $\mathfrak{M}$ that satisfy the 'domain-formula' $\delta(x)$, and by letting the interpretations of the primitive terms $R_{i}$ of $\mathcal{L}_{\mathrm{S}}$ be the $n$-tuples of objects in the domain of $\mathfrak{M}$ that satisfy their translation formulas $\varphi_{R_{i}}\left(x_{1}, \ldots, x_{n}\right)$. Let us call the model $\mathfrak{M}^{\tau}$ that arises in this way from $\mathfrak{M}$ the structure that is induced by the translation $\tau$. Using the notion of a sentence $\varphi$ being true in a structure $\mathfrak{M}$ (in short, $\mathfrak{M} \models \varphi$ ), we can then define the notion of relative semantic interpretability as follows 42

Definition. A translation $\tau$ is called a semantic interpretation of $\boldsymbol{S}$ in $\boldsymbol{T}$ if for each $\mathcal{L}_{T^{-}}$structure $\mathfrak{M}$, such that $\mathfrak{M} \models \boldsymbol{T}$, we have $\mathfrak{M}^{\tau} \models \boldsymbol{S}$. A theory $\boldsymbol{S}$ is semantically interpretable in $\boldsymbol{T}$ if there is a semantic interpretation of $\boldsymbol{S}$ in $\boldsymbol{T}$.

Just like syntactic interpretability, semantic interpretability is a relation between theories. And this is as it should be. Once again, Hilbert is quite explicit about the

\footnotetext{
${ }^{42}$ Compare again 38 for a closer discussion of this notion for the first-order case.
} 
fact that his proofs establish the consistency of a certain set of statements, given the consistency of another theory. However, in contrast to the notion of syntactic interpretability, we now make explicit use of semantic vocabulary. That is, the notion of a structure for a certain language and of a sentence being true in a structure are vital for this conception. Given this reading, then, how exactly do we prove the consistency of a theory $\mathbf{S}$, given the consistency of another theory $\mathbf{T}$ in terms of the notion of semantic interpretability? The basic idea seems natural enough: since $\mathbf{T}$ is assumed to be consistent, there should be some structure $\mathfrak{M}$ in which the axioms of $\mathbf{T}$ are true. If, moreover, we assume that $\mathbf{S}$ is semantically interpretable in $\mathbf{T}$, then the structure $\mathfrak{M}^{\tau}$ (which is induced by a certain translation $\tau$ ) will be a model of $\mathbf{S}$. So $\mathbf{S}$ is satisfiable and therefore consistent as well 43

Now, Hilbert's procedure in his Foundations can be understood as exactly doing this. According to this interpretation, what Hilbert is doing in, say, his consistency proof for Euclidean geometry, goes as follows: Since he assumes that the background theory $\mathbf{A}$ of analysis is consistent, there is a model $\mathfrak{A}$ satisfying all the axioms of analysis, i.e. his axiom system for real numbers presented in [19]. Using a certain translation $\tau$, Hilbert then constructs from the objects and relations of $\mathfrak{A}$ a new structure $\mathfrak{A}^{\tau}$ for the language of Euclidean geometry. That is, the translation of geometrical statements into statements of analysis induces a new and purely 'analytic' model that satisfies (in the modern sense of the term) the axioms of Euclidean geometry EG. EG is thus shown to be semantically interpretable in the theory of real numbers A. It follows that EG is satisfiable, and, therefore, consistent as well. The question is, once again: Is there any textual evidence for such a reading in Hilbert's subsequent work? And again, there are indeed passages to be found in his later writings that suggest that Hilbert (with hindsight) did understand the $\mathrm{C} \& \mathrm{I}$ proofs in Foundations in such a way. For instance, in his lecture notes 'Grundlagen der Mathematik' from (1921/22) (published in [21]), Hilbert surveys two methods to prove the consistency of an axiomatic theory which he calls the 'method of presentation' ('Methode der Aufweisung') and 'method of reduction' ('Methode der Zurückführung'). The first method is arguably model-theoretic in

\footnotetext{
${ }^{43}$ Note that, in general, consistency implies the existence of a model only if $\mathbf{T}$ is a first-order theory. Here, the completeness theorem guarantees that every consistent set of statements has a model. However, due to the incompletability of higher-order logic, consistency does not entail satisfiability in a higher-order setting. Now, Hilbert is pretty clear about the fact that, in his C \& I proofs, his assumption of the consistency of the background theory is to be understood prooftheoretically. (See e.g. [17, §8], where Hilbert starts his investigation of the mutual independence of his axioms with the remark that 'in fact, it may be shown that none of [the axioms] can be deduced from the remaining ones by any logical process of reasoning'.) So, on the current reconstruction of his proofs, Hilbert is either wrongly assuming that the consistency of a theory implies the existence of a model even in a higher-order setting, or his notion of consistency is ambiguous between prooftheoretical consistency and satisfiability after all. Another option, suggested by a referee, is that in his C \& I proofs Hilbert simply assumes e.g. the existence of the real numbers and submodels thereof. Thus, on this reading, Hilbert assumes that the axioms for the real numbers are true for their intended model, and hence, consistent. But this seems to reverse the order of proof. Again, officially, Hilbert is quite explicit about the fact that he only assumes consistency, (officially) understood proof-theoretically. So all of this just strengthens our main argument: that a modeltheoretic reading of Hilbert's C \& I proofs is not as straightforward as it may seem.
} 
character in the modern sense of the term. It consists, essentially, in the construction of a finitary model of an axiomatic theory ${ }^{44}$

The method of reduction is more important in the present context given that it underlies Hilbert's C \& I proofs in Foundations ${ }^{45}$ This approach is arguably also semantic in character. It does not consist in the direct construction of a model, however, but rather in the semantic interpretation of one theory in another theory in the above sense. Hilbert describes this approach as follows:

Every geometrical statement can be expressed in the language of analytic geometry and is presented here as an arithmetical statement whose truth or falsity is determined on the basis of the arithmetical axioms (which we presuppose here). It follows from these remarks that the truth or falsity of the sentence also has to be directly determined by the axioms of geometry. [21, p. 444, our emphasis]

Several pages later he gives the following characterization:

The common principle consists in the fact that, in order to show that a system of axioms is consistent, one presents objects within analysis between which the relations stipulated in the axioms hold. [21, p. 462, our emphasis]

The important point to note about these passages is the semantic terminology (in particular, the notions of 'truth' and 'holding of') used to describe this kind of intertheoretic reduction. The same terminology is also employed in Hilbert's discussion of the method of finitary model constructions.

While the above passages still leave room for ambiguity between a semantic and a syntactic reading of modeling, there are other passages in Hilbert's retrospective discussion of his metatheoretical work on Euclidean geometry that paint a decidedly semantic picture. For instance, again in the Foundations of Mathematics from 1934, Hilbert gives the following characterization of the 'method of arithmetization' used in the independence proofs of his Foundations from 1899:

We are looking for an axiom system $\mathfrak{A}$ with the following properties: The structure of $\mathfrak{A}$ has to be comprehensible to such an extent that we can prove its consistency [...] [in terms of a direct proof]. $\mathfrak{A}$ has to be sufficiently comprehensive in the following sense. For an axiom system $\mathfrak{B}$ of the disciplines of geometry or physics, and from the assumed satisfaction of $\mathfrak{A}$ in a system of objects and relations $\mathfrak{S}$, we have to be able to derive the satisfaction of $\mathfrak{B}$ by individuals or complexes of individuals from $\mathfrak{S}$, and whose basic relations are predicates formed from the basic relations of $\mathfrak{S}$ by logical operations. 22, p. 18]

This passage strongly indicates a reconstruction of Hilbert's C \& I proofs in terms of semantic interpretability. All the ingredients are there: we assume some axiomatic 'background' theory, viz. analysis; we furthermore assume that this axiom system is satisfied ('erfüllt') by a certain 'system of objects and relations' (i.e. a 'model') and

\footnotetext{
${ }^{44}$ Hilbert illustrates the method in terms of a simple two-valued numerical model of an axiom system of propositional logic in [21, pp. 436-440]

${ }^{45}$ Compare Hilbert on this point: 'This method finds particularly fruitful applications in the axiomatic investigation of Euclidean geometry [...]' [21, p. 440]
} 
construct from this model the objects and relations of another model that satisfies the axioms of geometry ${ }^{46}$ Put differently, according to the account outlined here, the consistency of a geometrical theory is established by the construction of an internal model of this theory in the standard interpretation of the theory of analysis.

If Hilbert's method is understood in this way, we attribute to him an essentially modern model-theoretic understanding of the notions of 'satisfiability' or 'validity' of a set of statements in a structure ${ }^{47}$ More specifically, the key observation here is that we think of him as employing a general satisfaction relation between structures and statements. We have to assume that Hilbert had an intuitive understanding of what it means that an arbitrary structure for a given language satisfies a given sentence (or set of sentences) of this language. Of course, we don't have to assume that Hilbert had an idea of how to define such a relation. Tarski's formal work on truth was still a long way down the road. In their Foundations of Mathematics of 1934, Hilbert and Bernays do not mention Tarski's work on truth, and, obviously, Hilbert cannot be assumed to have known in 1899 how to define truth and, a fortiori, truth in a model ${ }^{48}$ At any rate, if correct, interpreting Hilbert as implicitly employing the notion of semantic interpretability would seem to force us to attribute to Hilbert an informal conception of a general satisfaction relation.

Notice that this model-theoretic reading is also motivated by Hilbert's own, albeit brief, discussion (presented above in section 2) of the semantic justification of the principle of duality. Recall that duality can be expressed as the fact that a statement in the language of projective geometry is true in a projective plane $\mathfrak{P}$ if and only if its dual statement is also true in that plane. As we saw, proving duality for a particular projective plane $\mathfrak{P}$ requires the construction of the dual structure $\mathfrak{P}^{d}$ and showing that it is isomorphic to structure $\mathfrak{P}$. The principle of duality then immediately follows from the isomorphism lemma. Indeed, it is precisely the isomorphism lemma that Hilbert seems to allude to in his remark written in response to Frege:

But it is surely obvious that every theory is only a scaffolding or schema of concepts together with their necessary relations to one another, and that the basic elements can be thought of in any way one likes. [...] In other words: any theory can always be applied to infinitely many systems of basic elements. One only needs to apply a reversible oneone transformation and lay it down that the axioms shall be correspondingly the same for the transformed things. This circumstance is in fact frequently made use of, e.g. in the principle of duality, etc., and I have made use of it in my independence proofs. (9, pp. 40-41])

This passage can be read as an early formulation of the isomorphism lemma: any two

\footnotetext{
${ }^{46}$ We have slightly modified the translation given in 22 in order to stay as close as possible to the German original. The translators of [22] paraphrase the passage in explicitly model-theoretic terms, translating e.g. 'die vorausgesetzte Erfüllung' by 'assumed existence of a model'.

${ }^{47}$ See [16, pp. 459-460] and [36, pp. 227-230] for such a model-theoretic interpretation of Hilbert's semantic terminology. Hallett, in [16], remains neutral between the purely syntactic and the modeltheoretic reading Hilbert's method of modeling.

${ }^{48}$ Tarski's celebrated paper 34] on the concept of truth in formalized languages was first published in Polish in 1933 and in 1935 in German.
} 
systems (with domains of different objects) are indiscernible as models of the theory if there exists a 1-1 mapping between them that preserves the structural properties expressed in the axioms. This semantic assumption seems to justify, in Hilbert's view, the use of analytic models of Euclidean geometry in his metatheoretic proofs.

\subsection{Consistency and arithmetical truth}

In light of the preceding remarks, one could infer that Hilbert understood the methods used in the Foundations in an essentially modern, model-theoretic way. According to this view, one and the same axiomatic theory can be interpreted by various different models and Hilbert uses such models (constructed from an assumed model for analysis) to establish his $\mathrm{C} \& \mathrm{I}$ results ${ }^{49}$

However, such an attribution of a modern model-theoretic conception of formal languages to Hilbert does not seem to be forced after all. One can think of a third way to interpret his method of modeling that is semantic in character but not in the modern model-theoretic sense of the term. The crucial observation here is this: Let us assume, as above, that we are given the intended model $\mathfrak{A}$ for the theory of analysis. Then, given some suitable translation $\tau$ of the language of geometry $\mathcal{L}_{\mathbf{E G}}$ in the language of analysis $\mathcal{L}_{\mathbf{A}}$, we can construct an analytic model $\mathfrak{A}^{\tau}$ of the geometrical theory EG. By definition, for any statement $\varphi$ in $\mathcal{L}_{\mathbf{E G}}$, we then have 50

$$
\mathfrak{A}^{\tau} \models \varphi \text { if and only if } \mathfrak{A}=\varphi^{\tau}
$$

So a geometrical sentence $\varphi$ is true in $\mathfrak{A}^{\tau}$ just in case its translation $\varphi^{\tau}$ is true in the original model of analysis 51 Now, the upshot of this is that this allows us to explain away the apparent model-variation in Hilbert's C \& I proofs. We might interpret Hilbert's method of 'modeling' in such a way that, in fact, he was only concerned with the right-hand-side of the equivalence as a way to 'simulate' genuine model-theoretic reinterpretation of the geometrical language $\mathcal{L}_{\mathbf{E G}}$. The underlying idea of Hilbert's method can be captured by the following definition:

Definition. A translation $\tau$ is called a semantic interpretation* of $\boldsymbol{S}$ in $\boldsymbol{T}$ if for

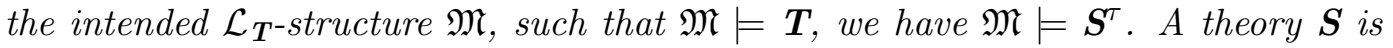
semantically interpretable* in $\boldsymbol{T}$ if there is a semantic interpretation* of $\boldsymbol{S}$ in $\boldsymbol{T}$.

To repeat, the idea here is that a theory $\mathbf{S}$ is interpretable in $\mathbf{T}$ if the translations of all $\mathbf{S}$-axioms are true in the intended model of $\mathbf{T}$. It follows that, on this conception, attributing to Hilbert the notion of various 'models' satisfying a given set of statements of a given language is not forced after all. Concerning his C \& I proofs in his Foundations more specifically, all we have to attribute to him is that he has

\footnotetext{
${ }^{49}$ See, for instance, [23, pp. 6-7] for such an interpretation.

${ }^{50}$ Note the similarity to the semantic justification of the semantic version of the principle of duality given earlier. Here too, given some projective plane $\mathfrak{P}$, we can always form the dual structure $\mathfrak{P}^{d}$ which is induced by the canonical translation $d$ which maps the word 'point' to 'line' and 'line' to 'point'. And here again we have $\mathfrak{P}^{d}=\varphi$ iff. $\mathfrak{P} \models \varphi^{d}$.

${ }^{51}$ Compare [25, §5.1] for a detailed treatment of model-theoretical relativization of this form.
} 
an intuitive grasp of the concept of arithmetic truth, i.e. truth in the standard model $\mathfrak{A}$ of the theory of analysis stated in [19. So whenever Hilbert 'reinterprets' a set of statements in the language of Euclidean geometry, what he actually does is to provide a syntactic translation that makes sure that the translation of each sentence in this set is a true arithmetical statement. According to this reconstruction, then, no genuine 'model-variation' is necessary to understand his C \& I proofs, and thus no model theory in the modern sense of the term. Neither the language of analysis nor the language of geometry are actually reinterpreted here. Their non-logical vocabulary is still tied to a fixed semantic interpretation ${ }^{52}$

Hilbert's scattered remarks on the 'method of arithmetization' at use in his geometrical C \& I proofs can be interpreted in this proto-semantic way. Notice, in particular, that in several of the passages cited above, Hilbert explicitly mentions the translation of geometrical into true arithmetical sentences, e.g.

Every geometrical statement can be expressed in the language of analytic geometry and is presented as an arithmetical statement here whose truth or falsity is determined on the basis of the arithmetical axioms (which we presuppose here). It follows from these remarks that the truth or falsity of the sentence also has to be directly determined by the axioms of geometry. [21, p. 444, our emphasis]

The method described here can be understood as a way to represent geometrical truth - that is, the truth of geometrical statements - in terms of arithmetical truth which, in turn, is presupposed here as a primitive notion. This is not to say that Hilbert was in any way interested in reducing geometrical truth to arithmetical truth in a foundational sense of the term. The arithmetization of geometry is conceived here as a metatheoretic tool that allows Hilbert to show the consistency of geometrical statements by translating them into true arithmetical statements. ${ }^{53}$ What such an interpretation of Hilbert's methodology in Foundations essentially comes down to is to attribute to him a substitutional conception of satisfaction with respect to some intended background model $\mathfrak{A}$ for analysis. A sentence $\varphi$ of the language of Euclidean geometry, for instance, is true with respect to a translation

\footnotetext{
${ }^{52}$ Notice that this reading is consistent also with Hilbert's independence proofs where analysis does not come into play. These proofs can all be understood as employing the same method. Consider e.g. the case of the axiom of parallels: what Hilbert is doing, on the current understanding, is to start with the standard model $\mathfrak{G}$ of ordinary Euclidean geometry; then he comes up with a translation $\tau$ such that the translation of the negation of the axiom of parallels is true in this standard model. Once again, we only have to assume that the notion of a geometrical sentence being true in the 'standard model' of Euclidean geometry is understood. No 'model variation' in the modern sense of the term is needed.

${ }^{53}$ As an anonymous referee pointed out, this reconstruction has the drawback that it becomes hard to understand the relative character of Hilbert's C \& I proofs. If, as suggested in this reconstruction, Hilbert simply assumes (as a matter of faith, as it were) that analysis is true, then his C \& I proofs establish consistency or independence relative to the truth of analysis, not its consistency. It's hard to see, then, how this would not result in absolute C \& I proofs, rather than relative C \& I proofs. Of course, if the axioms of analysis are true, they are a fortiori consistent. But, as mentioned in footnote 43 , this seems to reverse the order of proof. Hilbert officially only assumes the consistency of analysis, not its truth in some or other interpretation. Again, this seems to reinforce our argument that a semantic reading of Hilbert's C \& I proofs has its problems.
} 
$\tau$ if its substitution instance $\varphi^{\tau}$ is a true arithmetical sentence (that is, true in the standard model of analysis). Thus, according to this view, rather than introducing model-theoretic reasoning in geometry, Hilbert can be thought to have introduced in Foundations a systematic way of replacing geometrical by primitive arithmetical truth.

\section{Conclusion}

This paper addressed the question how metatheoretic reasoning had emerged in nineteenth-century geometry. In particular, our focus here was on two specific developments, namely debates on the proper justification of the principle of duality in projective geometry (in work by Poncelet, Gergonne, and Pasch) on the one hand and Hilbert's metatheoretic study of Euclidean geometry in [17] on the other hand. We showed that there are two conceptually distinct ways to think about the principle of projective duality: the first approach is essentially 'model-theoretic' in character and explains duality in terms of structure-preserving mappings between geometrical systems. As we argued, Poncelet's work on reciprocal figures and polar transformations can be considered an early and prototypical formulation of this semantic approach to duality. According to the second, 'proof-theoretic' approach, duality is explained in terms of theorem-preserving translations between statements of the language of projective geometry. This account also has roots in nineteenth-century mathematics, in particular, in Pasch's pioneering work on axiomatic projective geometry in [29].

A central objective in the paper was to see how these two ways of justifying duality are related to Hilbert's independence and consistency results in the Foundations. As we saw, Hilbert (at least at one point, in his famous letter to Frege), made an explicit connection between the semantic or mapping-based conception of duality and his own C \& I proofs. This seems to give further confirmation of the received view expressed by Hinitkka and others that Hilbert's method of modeling is genuinely semantic and that his Foundations mark a cornerstone in modern model-theoretic reasoning. However, a closer reading of Hilbert's actual proofs of the independence of axioms and the consistency of (fragments of) his axiom system in [17 has shown that such a reading is not forced. Instead of being based on the semantic reinterpretation of the language of geometry, Hilbert's results might equally well be understood in terms of the notion of syntactic interpretability.

We are thus left with a mixed assessment of Hilbert's early metatheoretic approach. More specifically, what our study points to is a systematic ambiguity in Hilbert's axiomatic work between a syntactic (or proof-theoretic) and a semantic (or modeltheoretic) understanding of his method of modeling. This ambiguity is not altogether surprising given the fact that the Foundations were written more than three decades prior to the 'semantic turn' in Tarski's and Carnap's work and thus long before a clear syntax/semantics distinction was drawn in logical theory. Moreover, as the case of duality in projective geometry has shown, a similar parallelity of methods that we would today classify as model-theoretic and proof-theoretic is already present in 
the immediate geometrical prehistory of Hilbert's axiomatic work.

Somewhat more surprising is the fact that Hilbert's understanding of his C \& I proofs remains ambivalent even in his retrospective discussions of Foundations. As we have shown, there are several places in Hilbert's foundational work from the 1920s and 1930s in which his method of arithmetical modeling in geometry is readdressed. Here again, Hilbert's discussion of his consistency proofs and the general method underlying them fluctuates between a semantic and a purely syntactic reading. To show this in more precise terms, we developed three possible logical reconstructions of his results based on the notions of syntactic and semantic interpretability. Each of these reconstructions can be supported by textual evidence from Hilbert's Foundations as well as his later writings on the subject. Moreover, these logical reconstructions also allow us to see more clearly the connections between Hilbert's metatheoretical results and the preceding work on the justification of duality. The proof of relative consistency in terms of the notion of syntactic interpretability is closely related to the proof-theoretic justification of duality. In turn, thinking about Hilbert's consistency proofs in terms of semantic interpretability makes them comparable to the semantic treatment of duality sketched above.

Where does the present study leave us in our understanding of Hilbert's foundational work and its place in the history of modern logic? We will restrict ourselves to two brief remarks that might be developed further in future research. The first one concerns the proper assessment of Hilbert's methodological contributions in the Foundations. What the present study has hopefully shown is that the dominant understanding of Hilbert's book as a birth-place of modern model theory should be taken with a grain of salt. While it is true that Hilbert employs terminology that looks semantic from a modern point of view, it is not at all clear that he also understood it in a sense that we today would classify as model-theoretic. As Hilbert's subsequent discussions in his early axiomatic work in geometry show, he in fact might have thought about his $\mathrm{C} \& \mathrm{I}$ results in a purely syntactic or proof-theoretic way. In any case, the present findings suggest that a more refined interpretation of Hilbert's early metatheoretic work is needed in order to do justice to the subtleties of his approach.

The second remark concerns the significance of Hilbert's foundational work in the development of model theory and modern logic more generally. We believe that the more nuanced interpretation of Hilbert's metatheoretic approach in Foundations presented here could form the basis for a critical re-assessment of his contributions visà-vis the work of his contemporaries as well as of Hilbert's actual influence on later logicians. This concerns, in particular, the notorious Frege-Hilbert debate on the proper understanding of axiom systems, mathematical definitions, and metamathematical reasoning 54 It also concerns various points of contact between Hilbert's metatheoretical approach and subsequent work by Carnap, Gödel, and Tarski on the logical (i.e. type-theoretic) explication of metatheoretical concepts such as truth

\footnotetext{
${ }^{54}$ On the Frege-Hilbert controversy see, in particular, 5] and the references listed there. For more recent and detailed studies of Frege's understanding of metatheoretical concepts see [4] and [7.
} 
in a model, logical consequence, and relative consistency ${ }_{55}^{55}$

\section{Acknowledgements}

Research on this article was partly funded by the Austrian Science Fund (FWF) (Project numbers P 27718-G16 and P 30448-G24). Earlier drafts of this paper were presented at the Logik Café Colloquium in Vienna, the MCMP Colloquium in Munich, the third international meeting of the APMP in Paris in 2016 as well as at the conference Model Theory: Philosophy, Mathematics and Language in Munich in 2017. We would like to thank the members of the respective audiences as well as Wilfried Sieg, Eduardo Giovannini, Dirk Schlimm, Erich Reck, Timo Beringer, and Esther Ramharter for helpful discussions and valuable feedback. We also wish to thank two anonymous referees for their suggestions that have helped to improve this paper.

\section{References}

[1] K. Andersen. The Geometry of an Art - The History of the Mathematical Theory of Perspective from Alberti to Monge. Berlin Heidelberg: Springer, 2007.

[2] A. Arana and P. Mancosu. On the Relationship between Plane and Solid Geometry. The Review of Symbolic Logic, 5(2):294-353, 2012.

[3] R. Baldus. Zur Axiomatik der Geometrie. Über Hilberts Vollständigkeitsaxiom. Mathematische Annalen, 100:321-333, 1928.

[4] P. Blanchette. Frege's Conception of Logic. Oxford: Oxford University Press, 2012.

[5] P. Blanchette. The Frege-Hilbert Controversy. In E. N. Zalta, editor, The Stanford Encyclopedia of Philosophy (Spring 2014 Edition). http://plato.stanford.edu/archives/spr2014/entries/frege-hilbert/, 2014.

[6] H.M.S. Coxeter. Projective Geometry. Berlin: Springer, 2nd edition, 1987.

[7] G. Eder. Frege's 'On the Foundations of Geometry' and Axiomatic Metatheory. Mind, 125(497):5-40, 2016.

[8] J. V. Field and J. J. Gray. The Geometrical Work of Girard Desargues. New York: Springer, 1987.

[9] G. Frege. Philosophical and Mathematical Correspondence. Oxford: Blackwell Publishers, 1980.

\footnotetext{
${ }^{55}$ Compare 31] for a comparative study of work on these notions in the course of the 1920s and 1930s.
} 
[10] E. Giovannini. Bridging the Gap between Analytic and Synthetic Geometry: Hilbert's Axiomatic Approach. Synthese, 193:31-70, 2016.

[11] J. Gray. Ideas of Space: Euclidean, Non-Euclidean, and Relativistic. Oxford: Oxford University Press, 1989.

[12] J. Gray. Worlds out of Nothing - A Course in the History of Geometry in the 19th Century. New York: Springer, 2007.

[13] J. Gray. Plato's Ghost: The Modernist Transformation of Mathematics. Princeton: Princeton University Press, 2008.

[14] M. Hallett. Hilbert's Axiomatic Method and the Laws of Thought. In Alexander George, editor, Mathematics and Mind, pages 158-200. Oxford: Oxford University Press, 1994.

[15] M. Hallett. Reflections on the Purity of Method in Hilbert's Grundlagen der Geometrie. In P. Mancosu, editor, The Philosophy of Mathematical Practice, pages 198-255. Oxford: Oxford University Press, 2008.

[16] M. Hallett. Frege and Hilbert. In M. Potter and T. Ricketts, editors, The Cambridge Companion to Frege, pages 413-464. Cambridge: Cambridge University Press, 2010.

[17] D. Hilbert. Grundlagen der Geometrie. Leipzig: Teubner, 1968, 10th edition, An English translation is available as Foundations of Geometry, L. Unger (ed.), La Salle: Open Court Press, 1971.

[18] D. Hilbert. Mathematische Probleme. Nachrichten von der königlichen Gesellschaft der Wissenschaften zu Göttingen, mathematisch-physikalische Klasse, (3):253-296, 1900.

[19] D. Hilbert. Über den Zahlbegriff. Jahresbericht der deutschen MathematikerVereinigung, (8):180-185, 1900.

[20] D. Hilbert. David Hilbert's Lectures on the Foundations of Geometry 18911902. Springer-Verlag Berlin Heidelberg, 2004.

[21] D. Hilbert. David Hilbert's Lectures on the Foundations of Arithmetic and Logic 1917-1933. Berlin Heidelberg: Springer, 2013.

[22] D. Hilbert and B. Bernays. Grundlagen der Mathematik, volume 1. Berlin: Springer, An English translation is available as Foundations of Mathematics I, C.-P. Wirth (ed.), London: College Publications, 20111934.

[23] J. Hintikka. On the Development of the Model-theoretic Viewpoint in Logical Theory. Synthese, 77(1):1-36, 1988.

[24] J. Hintikka. What is the Axiomatic Method? Synthese, 183(1):69-85, 2011. 
[25] W. Hodges. Model Theory. Cambridge: Cambridge University Press, 1993.

[26] M. Kline. Mathematical Thought from Ancient to Modern Times. New York: Oxford University Press, 1972.

[27] P. Lindström. Aspects of Incompleteness. Berlin: Springer, 1997.

[28] E. Nagel. The Formation of Modern Conceptions of Formal Logic in the Development of Geometry. Osiris, 7:142-223, 1939.

[29] M. Pasch. Vorlesungen über neuere Geometrie. Leipzig: Teubner, 1882.

[30] V. Poncelet. Traité des propriétés projectives des figures. Paris Gauthier-Villars, 1822.

[31] G. Schiemer and E. Reck. Logic in the 1930s: Type Theory and Model Theory. Bulletin of Symbolic Logic, 19(4):433-472, 2013.

[32] D. Schlimm. Pasch's Philosophy of Mathematics. Review of Symbolic Logic, $3(1): 93-118,2010$.

[33] W. Sieg. The Ways of Hilbert's Axiomatics: Structural and Formal. Perspectives on Science, 22(1):133-157, 2014.

[34] A. Tarski. Der Wahrheitsbegriff in den formalisierten Sprachen. Studia Philosophica, 1:261-405, 1935.

[35] M. Toepell. Über die Entstehung von David Hilberts Grundlagen der Geometrie. Göttingen: Vandenhoeck \& Ruprecht, 1986.

[36] R. Torretti. Philosophy of Geometry from Riemann to Poincaré. Dordrecht/Boston/London: D. Reidel, 1978.

[37] O. Veblen and J.W. Young. Projective Geometry. New York: Ginn and Company, 1938.

[38] A. Visser. An Overview of Interpretability Logic. Logic Group Preprint Series, $174,2008$.

Author information:

Günter Eder: Department of Philosophy, University of Salzburg, Franziskanergasse 1 A-5020 Salzburg, Austria

Georg Schiemer, Department of Philosophy, University of Vienna, Universitätsstraße 7 A-1010 Vienna, Austria 